SU-ITP-08/36

\title{
Supersymmetric Heterotic Action out of M5 Brane
}

\author{
Jaemo Park $1,2,2$, and Woojoo Sim叶 \\ ${ }^{1}$ Department of Physics, POSTECH \\ Pohang 790-784, Korea \\ ${ }^{2}$ Postech Center for Theoretical Physics (PCTP), POSTECH \\ Pohang 790-784, Korea \\ ${ }^{3}$ Department of Physics, Stanford University \\ Stanford, CA 94305-4060, USA
}

\begin{abstract}
Generalizing the work by Cherkis and Schwarz [1], we carry out the double dimensional reduction of supersymmetric M5 brane on K3 to obtain the supersymmetric action of heterotic string in 7-dimensional flat space-time. Motivated by this result, we propose the supersymmetric heterotic action in 10-dimensional flat space-time where the current algebra is realized in a novel way. We explicitly verify the $\kappa$-symmetry of the proposed action.
\end{abstract}

*jaemo@postech.ac.kr

†space@postech.ac.kr 


\section{Introduction}

Understanding of BPS objects in string theory and M theory shed great insight on the structure of string and $\mathrm{M}$ theory. The prominent example is D-brane in string theory, which facilitated great progress in understanding the nonperturbative aspects of string theory. In M-theory, the counterpart to D-brane is M2 and M5-brane. Certainly the better understanding of these BPS objects would lead to the enhanced understanding of M-theory but the status quo is something to be desired compared with the understanding of D-brane. In the current paper, we concentrate on the M5-brane and make use of the M5-brane action, in particular, for one application. One peculiar feature of M5-brane is that the worldvolume excitations contain self-dual 2-form fields. In order to write a DiracBorn-Infeld (DBI) type action, novel approaches are needed. In case the worldvolume is 6-d Minkowskian spacetime, one way is to give up the manifest Lorentz invariance and to write apparent non-covariant action with hidden Lorentz invariance [2]. Another way is to impose the self-duality via gauge symmetry with auxiliary fields [3]. In this formalism, after a suitable gauge fixing, the action is the same as the one proposed at ref. [2] as shown at ref. [4]. The supersymmetric action of the M5-brane in the flat background was constructed in ref. [4]. M5-brane action in arbitrary curved background was constructed in ref. [5] and its worldvolume field equations were developed in ref. [6, 7, 8].

It is shown at ref. [1] that M5-brane wrapping on K3 gives rise to the heterotic string in 7-dimension for the bosonic action. It is natural to consider the supersymmetric generalization of it. We explicitly construct the supersymmetric heterotic action in 10-d Minkowskian space motivated by the action derived from the M5-brane wrapping on K3. There are various constructions of kappa symmetric heterotic actions [9, 10, 11, 12]. In each of the cases, the realization of the chiral current algebra is different and it's interesting to show the equivalence of each approach.

The content of the paper is as follows. In section 2, we review the bosonic M5-brane wrapped on K3 [1]. In section 3, we extend the reduction to the case of the supersymmetric M5-brane action. The resulting action is the supersymmetric heterotic action in

7-dimensional flat space. Interesting point is that the lattice derived from the two forms of K3 has the signature of $(19,3)$. The zero modes of the self-dual two-form give rise to 22 scalars of the heterotic action. Among these scalars, only 3 scalars coming from $(0,3)$ part have the supersymmetric extension while the other 19 scalars have the same form as in the bosonic case. Although the dimensional reduction is carried out at the leading orders of the fermionic variables, it is sufficient to give the clue to write the heterotic action in 10-d. In section 4, we write down the supersymmetric heterotic action in 10-d flat space. To realize the current algebra, we have to introduce 16 scalars. From the exercise of the section 3 , we guess that the action of the scalar is the same as that of bosonic case. Indeed we can explicitly verify the kappa symmetry of the proposed heterotic action. In the appendix we 
present the details of the proof of the kappa symmetry.

\section{Reduction of the Bosonic M5-Brane:Review}

In this section, we review the bosonic M5-brane wrapped on K3, which is introduced in ref. [1]. The M5-brane action used here is the one introduced in ref. [3] where the worldvolume covariance is manifest. The action is given as 1

$$
\begin{aligned}
& \mathcal{L}_{1}=-\sqrt{-\operatorname{det}\left(G_{\mu \nu}+i \frac{\widetilde{H}_{\mu \nu}}{\sqrt{-G u^{2}}}\right)}, \\
& \mathcal{L}_{2}=-\frac{1}{4 u^{2}} \widetilde{H}^{\mu \nu} H_{\mu \nu \rho} u^{\rho} .
\end{aligned}
$$

The worldvolume fields of the action are $X^{M}$, the coordinates of the target 11-d spacetime, and $B_{\mu \nu}$, the chiral two-form whose field strength $H_{\mu \nu \rho}$ is self-dual. In addition, to make the general worldvolume covariance of the action manifest, an auxiliary scalar field $a$ is introduced as $u_{\mu}=\partial_{\mu} a$. In the action, the bosonic background $e_{M}^{\widehat{M}}$ couples to the worldvolume fields through the pullback

$$
G_{\mu \nu}=\partial_{\mu} X^{M} e_{M}^{\widehat{M}} \partial_{\nu} X^{N} e_{N}^{\widehat{N}} \eta_{\widehat{M} \widehat{N}}=\partial_{\mu} X^{M} \partial_{\nu} X^{N} g_{M N}
$$

which is the induced worldvolume metric $2 \widetilde{H}^{\mu \nu}$ in the action is defined as

$$
\widetilde{H}^{\mu \nu}=\frac{1}{6} \epsilon^{\mu \nu \rho \sigma \tau \lambda} H_{\rho \sigma \tau} u_{\lambda} .
$$

The dimensional reduction here is a double dimensional one, which means that we wrap the worldvolume on the compact part of the target manifold (K3 here). Then, denoting the coordinates of $\mathrm{K} 3$ by $\sigma^{i}$, we can take the static gauge $X^{i}=\sigma^{i}$ by using the worldvolume covariance of the action, such that $\sigma^{\mu}=\left(\xi^{\alpha}, \sigma^{i}\right)$ and $X^{M}=\left(X^{m}, \sigma^{i}\right)$ where $\xi^{\alpha}$ are the world sheet coordinates of the resulting heterotic string action.

In reduction, we take the zero modes of the fields on K3. For the chiral two-form $B_{\mu \nu}$, the zero modes are the harmonic forms on K3. However, the harmonic zero-form $B_{\alpha \beta}$ and the harmonic one-form $B_{\alpha i}$ on K3 do not contribute to the reduction because $\partial_{i} B_{\alpha \beta}=0$ and $B_{\alpha i}=0$. Then, the only contributing one is the harmonic two-form on K3,

$$
B_{i j}=\sum_{I=1}^{22} Y^{I}(\xi) b_{I i j}(\sigma),
$$

\footnotetext{
${ }^{1}$ We use the following indices for the coordinates of the related manifolds. For the M5-brane worldvolume and for the string worldsheet, $\mu, \nu, \cdots$ and $\alpha, \beta, \cdots$ are used, respectively. For the 11-d and the 7-d target spaces, $M, N, \cdots$ and $m, n, \cdots$ are used, respectively. We use $i, j, \cdots$ for K3 surface. Finally, hatted indices are used for the corresponding tangent spaces of the manifolds.

${ }^{2}$ The worldvolume indices in the action are raised and lowered by the induced metric.
} 
where $B_{i j}$ is expanded into the 22 linearly independent harmonic two-forms $b_{I i j}$ which form a basis of $\operatorname{Harm}^{2}(\mathrm{~K} 3, \mathbb{Z}) \cong \mathrm{H}^{2}(\mathrm{~K} 3, \mathbb{Z})$. Among $b_{I i j}$, three are self-dual and the rest are anti-self-dual.

Then, by using eq. (2.5) we find that the corresponding nonvanishing components of $H_{\mu \nu \rho}$ and $\widetilde{H}^{\mu \nu}$ are, respectively,

$$
H_{\alpha i j}=\sum_{I=1}^{22} \partial_{\alpha} Y^{I} b_{I i j}, \quad \widetilde{H}^{i j}=\sum_{I=1}^{22} \sqrt{h} \widetilde{Y}^{I}\left(* b_{I}\right)^{i j}
$$

where $h=\operatorname{det} h_{i j}, h_{i j}$ being the K3 metric, and $\tilde{Y}^{I}=\epsilon^{\alpha \beta} \partial_{\alpha} Y^{I} u_{\beta}$.

For the case of $X^{M}$ we can take $\partial_{i} X^{M}=\delta_{i}^{M}$ by using the static gauge $X^{i}=\sigma^{i}$. This gives the decomposition of induced metric as a block diagonal form $G_{\mu \nu}=\widetilde{G}_{\alpha \beta} \oplus h_{i j}$, where $\widetilde{G}_{\alpha \beta}$ is the world-sheet induced metric. (As usual, the target manifold is a product one $M_{7} \times \mathrm{K} 3, M_{7}$ being a 7 -dimensional flat spacetime, so that the metric becomes block diagonal: $g_{M N}=g_{m n} \oplus h_{i j}$.)

Then, inserting above nonvanishing components $H_{\alpha i j}, \widetilde{G}_{\alpha \beta}$ and $h_{i j}$ into the action and integrating over $\mathrm{K} 3$, the final form of the reduced action is given as

$$
S=-\sqrt{-\widetilde{G}} \sqrt{1+\frac{\widetilde{Y}^{I} M_{I J} \widetilde{Y}^{J}}{\widetilde{G} u^{2}}-\left(\frac{\widetilde{Y}^{I} L_{I J} \widetilde{Y}^{J}}{2 \widetilde{G} u^{2}}\right)^{2}}-\frac{\widetilde{Y}^{I} L_{I J} \partial_{\alpha} Y^{J} u^{\alpha}}{2 u^{2}},
$$

where

$$
L_{I J}=\int_{K 3} b_{I} \wedge b_{J}, \quad M_{I J}=\int_{K 3} b_{I} \wedge * b_{J} .
$$

Here the point is that the reduced action eq. (2.7) is the dual of the heterotic string action compactified on $T^{3}$. The key clue is given by the $Y^{I} b_{I i j}$ of eq. (2.5) and by the matrices $L$ and $M$ of eq. (2.8). In fact, $b_{I}$ of $\mathrm{K} 3$ are the basis of the self-dual lattice of signature $(19,3)$, which is unique up to isometries, so that $L$ is the matrix of inner products between the basis elements [13, 14, 15]. On the string side, as the heterotic string winds around $T^{3}$, there appear $16+3$ left-movers and 3 right-movers. These modes make the even self-dual lattice of signature $(19,3)$, so called Narain lattice. Thus, we can identify the Narain lattice with the lattice arising from the M5-brane wrapped on K3, with the 22 movers being $Y^{I}$. Moreover, $M$ can be interpreted as the matrix in which the moduli of the torus are encoded. In fact, the 7 -d action eq. (2.7) can be directly shown to be equal

to the heterotic action compactified on $T^{3}$ as in ref. [1]. Note that $Y^{I}$ are the usual scalars in the above action (2.7) but the additional gauge symmetry makes $Y^{I}$ to be chiral.

\section{Reduction of the Supersymmetric M5-brane}

Now, let us wrap the supersymmetric M5-brane on K3, by extending the bosonic case above, and see what the resulting 7 -d theory is. Here the fermionic coordinate $\theta$ of the 11-d 
superspace and its bilinears on K3 play the key role. We first review the supersymmetric M5-brane action, and then perform the reduction. In order to do it properly, we need the full supersymmetric M5-brane action in an arbitrary supergravity background and carry out the dimensional reduction. However we expect to obtain the heterotic action in the flat space after the dimensional reduction and the action should have the form

$$
\mathcal{L}=-\sqrt{-G} \sqrt{1+\cdots}-\epsilon^{\alpha \beta} \bar{\theta} \Gamma_{m} \partial_{\alpha} \theta \partial_{\beta} X^{m}+\cdots
$$

We can guess this from the Type I kappa symmetric action and bosonic part of the heterotic action. The structure we do not know is how the scalar degrees of freedom representing $E_{8} \times E_{8}$ current algebra are entered in the supersymmetric way. Thus our strategy is to start from the supersymmetric M5-brane action in the flat space and modify it in a minimal way so that it can describe M5-brane action in an arbitrary supergravity background in leading order in $\theta$. In fact, at the leading order of $\theta$, the fermionic part coincides with the flat case as shown by de Wit for M2-brane [16] and we expect the similar result for M5-brane. Then we carry out the dimensional reduction to see the structure of the current algebra parts of the supersymmetric heterotic action in 7-dimensional flat space. With that information, it is easy to guess the form of heterotic action in 10-dimensional flat space. We will write that action in section 4 and explicitly verify the kappa symmetry as well as supersymmetry.

\subsection{Supersymmetric M5-brane action}

To construct the supersymmetric M5-brane action, we need to extend the target space to the superspace with the coordinates $Z^{A}(\sigma)=\left(X^{M}, \theta^{a}\right)$, where $\theta(\sigma)$ is a 32-component Majorana spinor, an irreducible representation in 11-d. Then, together with the two-form field $B_{\mu \nu}$, both the bosonic and the fermionic fields in the theory have the same correct on-shell degrees of freedom, eight: five for $X^{M}$, three for $B_{\mu \nu}$; and eight for $\theta$, as expected for a brane of maximal supersymmetry. The auxiliary scalar field $a(\sigma)$ keeps its role as making the general covariance manifest. The concrete form of the action is [4, 5]

$$
S_{M 5}=\int d^{6} \sigma\left(\mathcal{L}_{1}+\mathcal{L}_{2}\right)+S_{W Z}
$$

where

$$
\begin{aligned}
\mathcal{L}_{1} & =-\sqrt{-\operatorname{det}\left(G_{\mu \nu}+i \frac{\widetilde{\mathcal{H}}_{\mu \nu}}{\sqrt{-G u^{2}}}\right)}, \\
\mathcal{L}_{2} & =-\frac{1}{4 u^{2}} \widetilde{\mathcal{H}}^{\mu \nu} \mathcal{H}_{\mu \nu \rho} G^{\rho \sigma} u_{\sigma}, \\
S_{W Z} & =\int\left(c_{6}+\frac{1}{2} H \wedge c_{3}\right) .
\end{aligned}
$$


Here, the worldvolume fields $Z^{A}(\sigma)$ and the 11-d supergravity backgrounds enters the action via the pullbacks

$$
\begin{aligned}
\Pi_{\mu}^{\widehat{A}} & =\partial_{\mu} Z^{A} E_{A}^{\widehat{A}} \\
c_{\mu \nu \rho} & =\partial_{\mu} Z^{C} \partial_{\nu} Z^{B} \partial_{\rho} Z^{A} C_{A B C}, \\
G_{\mu \nu} & =\Pi_{\mu}^{\widehat{M}} \Pi_{\nu}^{\widehat{N}} \eta_{\widehat{M} \widehat{N}}
\end{aligned}
$$

where $E_{A} \widehat{A}^{A}$ and $C_{A B C}$ are the supervielbein and the three-superform of the 11-d supergravity, respectively. In $S_{W Z}, c_{6}$ is the pullback of $C_{6}$ whose field strength is the dual of $d C_{3}$ :

$$
* d C_{3}=d C_{6}+\frac{1}{2} C_{3} \wedge d C_{3} .
$$

The two-form field $B_{\mu \nu}$ enters the action via the supersymmetrized field strength $\mathcal{H}$ :

$$
\mathcal{H}=d B-c_{3}, \quad \widetilde{\mathcal{H}}^{\mu \nu}=\frac{1}{6} \epsilon^{\mu \nu \rho \sigma \tau \lambda} \mathcal{H}_{\rho \sigma \tau} u_{\lambda}
$$

where $c_{3}$ is the pullback three-from in eq. (3.4).

Now, let us express the above pulled-back quantities by using the supervielbein $E_{A} \widehat{A}$ evaluated up to the 2 nd order of $\theta$ and the three-superform $C_{A B C}$ taken up to the leading order in $\theta$. Suppressing the gravitino and the 3 -form gauge field contributions, the components of $E_{A} \widehat{A}$ and $C_{A B C}$ are given as [16]

$$
\begin{aligned}
E_{M}^{\widehat{M}} & =e_{M}^{\widehat{M}}-\bar{\theta} \Gamma^{\widehat{M}} \omega_{M} \theta, & E_{M}^{\widehat{a}} & =\left(\omega_{M} \theta\right)^{\widehat{a}}, \\
E_{a}^{\widehat{M}} & =-\left(\bar{\theta} \Gamma^{\widehat{M}}\right)_{a}, & E_{a}^{\widehat{a}} & =\delta_{a}^{\widehat{a}}, \\
C_{M N P} & =0, & C_{M N a} & =\left(\bar{\theta} \Gamma_{M N}\right)_{a}, \\
C_{M a b} & =\left(\bar{\theta} \Gamma_{M N}\right)_{(a}\left(\bar{\theta} \Gamma^{N}\right)_{b)}, & C_{a b c} & \left.=\left(\bar{\theta} \Gamma_{M N}\right)_{(a}\left(\bar{\theta} \Gamma^{M}\right)_{b}\left(\bar{\theta} \Gamma^{N}\right)_{c}\right),
\end{aligned}
$$

where $e_{M}^{\widehat{M}}$ is the bosonic vielbein and $\omega_{M}=\frac{1}{4} \omega_{M}^{\widehat{P}} \widehat{Q} \Gamma_{\widehat{P} \widehat{Q}}$ is the spin connection. Here we take essentially the leading order expression in $\theta$ with minimal addition to make the partial derivative acting on $\theta$ covariant derivative.

Then, by using eq. (3.7), the components of the pullback vielbein $\Pi_{\mu}^{\widehat{A}}$ in eq. (3.4) are evaluated as

$$
\begin{aligned}
\Pi_{\mu}^{\widehat{M}} & =\partial_{\mu} X^{M} E_{M}^{\widehat{M}}+\partial_{\mu} \theta^{a} E_{a}^{\widehat{M}} \\
& =\partial_{\mu} X^{M} e_{M}^{\widehat{M}}-\bar{\theta} \Gamma^{\widehat{M}} \nabla_{\mu} \theta, \\
\Pi_{\mu}^{\widehat{a}} & =\partial_{\mu} X^{M} E_{M}^{\widehat{a}}+\partial_{\mu} \theta^{a} E_{a}^{\widehat{a}} \\
& =\nabla_{\mu} \theta^{\widehat{a}},
\end{aligned}
$$

where $\nabla_{\mu} \theta=\left(\partial_{\mu}+\omega_{\mu}\right) \theta$ and $\omega_{\mu}=\partial_{\mu} X^{M} \omega_{M}$, in which the worldvolume spin connection $\omega_{\mu}^{\widehat{\mu} \widehat{\nu}}$ (under static gauge) is encoded as well as the $S O(5)$ gauge connection [17]. 
In a similar manner, the pullbacks $c_{3}$ and $c_{6}$ are evaluated as

$$
\begin{aligned}
c_{3}= & \frac{1}{2} \bar{\theta} \Gamma_{M N} d \theta\left(d X^{M} d X^{N}+\bar{\theta} \Gamma^{M} d \theta d X^{N}+\frac{1}{3} \bar{\theta} \Gamma^{M} d \theta \bar{\theta} \Gamma^{N} d \theta\right) \\
c_{6}= & \bar{\theta} \Gamma_{M_{1} \cdots M_{5}} d \theta\left(\frac{1}{5 !} d X^{M_{1}} \cdots d X^{M_{5}}+\frac{1}{48} \bar{\theta} \Gamma^{M_{1}} d \theta d X^{M_{2}} \cdots d X^{M_{5}}\right) \\
& + \text { terms vanishing for the reduction on a 4-manifold, }
\end{aligned}
$$

where $d X^{M}=\partial_{\mu} X^{M} d \sigma^{\mu}$ and $d \theta=\nabla_{\mu} \theta d \sigma^{\mu}$. We emphasize again that there are higher order corrections in $\theta$ for an arbitrary background but by concentrating on the leading order corrections we get enough information to guess the heterotic action in 10-dimensional flat space. As we will see later, the resulting 7-dimensional string action obtained by leading order approximation is likely to be exact, though we will not try to prove its kappa symmetry.

The super M5-brane action in eq. (3.2) is invariant under the kappa transformations of the worldvolume fields [5, 6]

$$
i_{\kappa} \Pi^{\widehat{a}}=\delta_{\kappa} Z^{A} E_{A}^{\widehat{a}}=(1+\Gamma)_{\widehat{b}}^{\widehat{a}} \kappa^{\widehat{b}}, \quad i_{\kappa} \Pi^{\widehat{M}}=0, \quad \delta_{\kappa} \mathcal{H}=-i_{\kappa} d c_{3}, \quad \delta_{\kappa} a=0,
$$

where $i_{\kappa}$ denotes the pullback of the interior product on a background superform with respect to $\delta_{\kappa} Z^{A}$. In eq. (3.10), $\Gamma$ is determined to satisfy $\Gamma^{2}=1$ for the kappa invariance of the action:

$$
\Gamma=\frac{1}{\mathcal{L}_{1}}\left(\bar{\gamma}+\frac{1}{2 u^{2}} \widetilde{\mathcal{H}}^{\mu \nu} \gamma_{\mu \nu} \gamma^{\rho} u_{\rho}+\frac{1}{16 u^{2}} \epsilon_{\mu \nu \rho \lambda \tau \sigma} \widetilde{\mathcal{H}}^{\mu \nu} \widetilde{\mathcal{H}}^{\rho \lambda} \gamma^{\sigma \tau}\right)
$$

with $\gamma_{\mu}=\Gamma_{\widehat{M}} \Pi_{\mu}^{\widehat{M}}$ and $\bar{\gamma}=\gamma_{012345}$. In the flat background, the kappa variations above reduce to

$$
\delta_{\kappa} \theta=(1+\Gamma) \kappa, \quad \delta_{\kappa} X^{M}=-\delta \bar{\theta} \Gamma^{M} \theta, \quad \delta_{\kappa} \mathcal{H}_{\mu \nu \rho}=6 \delta \bar{\theta} \gamma_{[\mu \nu} \partial_{\rho]} \theta
$$

\subsection{Decomposition of a 11-d spinor}

Before we perform the reduction of the supersymmetric action, let us first examine the decomposition of 11-d spinor $\theta$ into 7-d and 4-d ones. The decomposition of 32 Majorana representation of $\operatorname{Spin}(10,1)$ under $\operatorname{Spin}(6,1) \times \operatorname{Spin}(4)$ is

$$
32=(8,2)+\left(8,2^{\prime}\right)
$$

where $\mathbf{2}$ and $\mathbf{2}^{\prime}$ are respectively positive and negative chirality Weyl spinors in 4-d, each of which is self-conjugate. As will be described in section 3.3, we take the 4-d spinors to be covariantly constant in the dimensional reduction. Then, since the holonomy group of K3 is $S U(2)$, which is a subgroup of $\operatorname{Spin}(4)=S U(2) \times S U(2)$, only one of $\mathbf{2}$ and $\mathbf{2}^{\prime}$ is covariantly constant according to whether K3 is self-dual or anti-self-dual. Therefore, taking $\mathbf{2}$ to be 
covariantly constant, only $(\mathbf{8}, \mathbf{2})$ of $\mathbf{3 2}$ contributes to the reduction on $\mathrm{K} 3$, and in 7 -d there are 16 real spinor degrees of freedom.

Now, let us express $(\mathbf{8}, \mathbf{2})$ in terms of irreducible representations of $\operatorname{Spin}(6,1)$ and $\operatorname{Spin}(4)$. Note first that the spinors of $\operatorname{Spin}(6,1)$ and $\operatorname{Spin}(4)$ cannot be the Majorana, but can be the symplectic Majorana (SM) [18, 19]. The SM representation is given as a $U S p(2)$ doublet, which is a pair of spinors $\psi_{1}$ and $\psi_{2}$ that satisfy

$$
\left(\psi_{A}\right)^{*}=B \epsilon^{A B} \psi_{B}, \quad B^{*} B=-1,
$$

with $\epsilon^{12}=-\epsilon^{21}=1$. (In the Majorana representation, $\psi^{*}=B \psi$ with $B^{*} B=1$.) Here, $B$ is a unitary matrix that relates $\Gamma_{\mu}$ to $\Gamma_{\mu}^{*}$, both of them being the representations of the Clifford algebra, as

$$
\Gamma_{\mu}^{*}=\eta B \Gamma_{\mu} B^{-1}, \quad \eta= \pm 1,
$$

where the sign of $\eta$ depends on the signature of the metric.

In the case of $\operatorname{Spin}(4)$, where the Weyl condition is available, the two spinors in eq. (3.14) can be the Weyl spinors of the same chirality. Therefore, the irreducible spinor representation of $\operatorname{Spin}(4)$ is symplectic Majorana-Weyl (SMW).

Now, considering above irreducible spinor representations in 7 -d and in 4 - $d$, we can express the 11 -d spinor $\theta$, which is $(\mathbf{8}, \mathbf{2})$ of $\mathbf{3 2}$, as

$$
\theta=\lambda_{1} \otimes \chi_{1}+\lambda_{2} \otimes \chi_{2},
$$

where $\lambda_{A}$ and $\chi_{A}$ denote $\operatorname{Spin}(6,1)$ and $\operatorname{Spin}(4)$ spinors satisfying the SM and the SMW (of positive chirality) conditions, respectively:

$$
\left(\lambda_{A}\right)^{*}=B_{7} \epsilon^{A B} \lambda_{B}, \quad\left(\chi_{A}\right)^{*}=B_{4} \epsilon^{A B} \chi_{B},
$$

where $B_{7}^{*} B_{7}=-1$ and $B_{4}^{*} B_{4}=-1$.

The SM representation as an $U S p(2)=S U(2)$ doublet implies that the 7 -d action has $S U(2)$ symmetry in the sense $S U(2)=S O(4) / S U(2)$ where $S O(4)$ and $S U(2)$ in r.h.s. are, respectively, the Lorentz and the holonomy group of K3. Then we need to assign the $S U(2)$ indices properly to $\lambda$ and $\chi$ according to their transformation properties under $S U(2)$ : lower indices to the ones transforming as fundamentals and upper indices to the ones transforming as complex conjugates. The result is

$$
\bar{\lambda}^{A}=\overline{\lambda_{A}} \quad\left(\lambda^{* A}=\left(\lambda_{A}\right)^{*}\right), \quad \bar{\chi}^{A}=\left(\chi_{A}\right)^{\dagger} \quad\left(\chi^{* A}=\left(\chi_{A}\right)^{*}\right) .
$$

This yields for 11-d spinor $\theta$ and its Dirac conjugate $\bar{\theta}$

$$
\begin{aligned}
\theta & =\lambda_{1} \otimes \chi_{1}+\lambda_{2} \otimes \chi_{2}, \\
\bar{\theta} & =\left(\left(\lambda_{1}\right)^{\dagger} \otimes\left(\chi_{1}\right)^{\dagger}+\left(\lambda_{2}\right)^{\dagger} \otimes\left(\chi_{2}\right)^{\dagger}\right) \widetilde{\Gamma}_{0} \otimes \widetilde{\Gamma}_{(5)} \\
& =\overline{\lambda_{1}} \otimes\left(\chi_{1}\right)^{\dagger}+\overline{\lambda_{2}} \otimes\left(\chi_{2}\right)^{\dagger} \\
& =\bar{\lambda}^{1} \otimes \bar{\chi}^{1}+\bar{\lambda}^{2} \otimes \bar{\chi}^{2},
\end{aligned}
$$


so that the $S U(2)$ invariant bilinear $\bar{\theta} \theta$ is expressed as

$$
\bar{\theta} \theta=\sum_{A, B} \bar{\lambda}^{A} \lambda_{B} \bar{\chi}^{A} \chi_{B}=\bar{\lambda}^{A} \lambda_{A} .
$$

Here we have used the decompositions of 11-d gamma matrices

$$
\Gamma_{m}=\widetilde{\Gamma}_{m} \otimes \widetilde{\Gamma}_{(5)}, \quad \Gamma_{i}=1 \otimes \widetilde{\Gamma}_{i}
$$

where $\widetilde{\Gamma}_{m}$ are the 7 -d gamma matrices while $\widetilde{\Gamma}_{i}$ the 4 -d ones with $\widetilde{\Gamma}_{(5)}=-\widetilde{\Gamma}_{78910}$.

To show the above expression of $\theta$ in eq. (3.16) is a consistent one, we should check $\theta$ can be a Majorana spinor with the expression. The complex conjugation of $\theta$ becomes

$$
\theta^{*}=\sum_{A} \lambda^{A *} \otimes \chi^{A *}=\sum_{A} B_{7} \epsilon^{A B} \lambda_{B} \otimes B_{4} \epsilon^{A C} \chi_{C}=\left(B_{7} \otimes B_{4}\right) \theta
$$

Then we can expect $B_{11}=B_{7} \otimes B_{4}$ to be the conjugation matrix in 11-d, satisfying the Majorana condition. In fact,

$$
\begin{aligned}
& B_{11}^{*} B_{11}=B_{7}^{*} B_{7} \otimes B_{4}^{*} B_{4}=-1 \otimes-1=1, \\
& B_{11}^{\dagger} B_{11}=B_{7}^{\dagger} B_{7} \otimes B_{4}^{\dagger} B_{4}=1,
\end{aligned}
$$

which shows that the 11-d Majorana condition, along with the 7-d SM and the 4-d SMW conditions, is satisfied.

In the tangent space bases of $\mathrm{K} 3$ and $M_{7}$, the gamma matrices and the corresponding conjugation matrices can be chosen as follows. In the case of K3, taking its coordinate to be $X^{7}, \cdots, X^{10}$,

$$
\widetilde{\Gamma}_{7,8,9}=\sigma_{1,2,3} \otimes \sigma_{2}, \quad \widetilde{\Gamma}_{10}=1 \otimes \sigma_{1}
$$

and for $M_{7}$,

$$
\widetilde{\Gamma}_{0,1,2}=\sigma_{1,2,3} \otimes \widetilde{\Gamma}_{(5)}, \quad \widetilde{\Gamma}_{3,4,5,6}=1 \otimes \widetilde{\Gamma}_{7,8,9,10}
$$

where $\widetilde{\Gamma}_{0}$ has an extra factor $i$. The conjugation matrices $B_{4}$ and $B_{7}$ that are compatible with the above gamma matrices can be

$$
B_{4}=i \sigma_{2} \otimes 1, \quad B_{7}=\sigma_{3} \otimes \sigma_{2} \otimes 1
$$

The covariantly constant spinors on K3 can be understood alternatively in the context of spin states that are created and annihilated by the gamma matrices associated with the Hermitian metric [20]. With the complex dimension being two, there are two creation (annihilation) operators $\widetilde{\Gamma}_{\bar{a}}\left(\widetilde{\Gamma}_{a}\right)$. Then, the two covariantly constant spinors are identified as the lowest state $|\Omega\rangle$ and the highest state $|\bar{\Omega}\rangle \sim \widetilde{\Gamma}_{12}|\Omega\rangle$, respectively. This is because these two spin states interact with the $U(1)$ part of the spin connection, which is trivial on K3 (and on any Calabi-Yau $n$-fold). 
On K3, the two spin states $|\Omega\rangle$ and $|\bar{\Omega}\rangle$ are of the same positive chirality, because the raising operator $\Gamma_{\bar{a}}$ flips the chirality and there are two such flips from $|\Omega\rangle$ to $|\bar{\Omega}\rangle$. Besides, since $\Gamma_{a}|\Omega\rangle=\Gamma_{\bar{a}}|\bar{\Omega}\rangle=0$, we can take the two SMW spinors $\chi_{1}$ and $\chi_{2}$ in eq. (3.17) to be $|\Omega\rangle$ and $|\bar{\Omega}\rangle$, respectively. This property is used in the following section to identify the spinor bilinears that appear in the reduction.

\subsection{Reduction of the supersymmetric action}

Now let us perform the double dimensional reduction of the supersymmetric M5-brane action in eq. (3.2) on K3. Before integrating over K3, let us first take the zero modes of the fields and find the components that contribute to the action. For the bosonic fields $B_{\mu \nu}$ and $X^{M}$ we can take their zero modes as in the bosonic case. For the fermionic field $\theta$, we can take its zero mode to satisfy

$$
\nabla_{i} \theta=\left(\partial_{i}+\frac{1}{4} \omega_{i}^{\widehat{\jmath} \widehat{ }} \Gamma_{\widehat{\imath}}\right) \theta=0
$$

i.e., covariantly constant on K3, since the spin connection $\omega_{i}^{\widehat{M} \widehat{N}}$ in the equation of motion reduces to $\omega_{i}^{\widehat{\imath} \widehat{\jmath}}$ in eq. (3.27) with the condition $\omega_{i}^{\widehat{m} \widehat{n}}=\omega_{i}^{\widehat{\jmath} \widehat{n}}=0$ given by the product manifold ansatz $e_{i}^{\widehat{m}}=\partial_{\alpha} e_{i}^{\widehat{\imath}}=0.3$

By using $\nabla_{i} \theta=0$ as well as the bosonic zero modes, we can evaluate the components of the pulled-back quantities in the action.

First, the components of pullback vielbein $\Pi_{\mu}^{\widehat{M}}$ in eq. (3.8) become

$$
\begin{array}{ll}
\Pi_{\alpha}^{\widehat{m}}=\partial_{\alpha} X^{m} e_{m}^{\widehat{m}}-\bar{\theta} \Gamma^{\widehat{m}} \nabla_{\alpha} \theta, & \Pi_{\alpha}^{\widehat{\imath}}=-\bar{\theta} \Gamma^{\widehat{\imath}} \nabla_{\alpha} \theta, \\
\Pi_{i}^{\widehat{m}}=e_{i}^{\widehat{m}}=0, & \Pi_{i}^{\widehat{\imath}}=e_{i}^{\widehat{\imath}} .
\end{array}
$$

Here, for $e_{\alpha}^{\widehat{\imath}}=\partial_{i} e_{m}^{\widehat{m}}=0, \nabla_{\alpha}=\partial_{\alpha}+\frac{1}{4} \omega_{\alpha}^{\widehat{m} \widehat{n}} \Gamma_{\widehat{m} \widehat{n}}, \operatorname{Spin}(6,1)$ spin connection being pulledback to 2 -d worldsheet. However, since we take $M_{7}$ to be Minkowskian, the connection is trivial so that $\nabla_{\alpha}=\partial_{\alpha}$.

Then, the components of $G_{\mu \nu}$ are given by using eq. (3.28) as

$$
G_{\alpha \beta}=\widetilde{G}_{\alpha \beta}+\bar{\theta} \Gamma_{i} \partial_{\alpha} \theta \bar{\theta} \Gamma^{i} \partial_{\beta} \theta, \quad G_{i j}=h_{i j}, \quad G_{\alpha i}=-\bar{\theta} \Gamma_{i} \partial_{\alpha} \theta
$$

where $\widetilde{G}_{\alpha \beta}=\eta_{\widehat{m} \widehat{n}} \Pi_{\alpha}^{\widehat{m}} \Pi_{\beta}^{\widehat{n}}$ is the induced metric of the 2 -d worldsheet in the flat 7 -d superspace background, and $h_{i j}$ is the metric of K3 surface.

Next, we get the components of $c_{3}$ in eq. (3.9) as

$$
c_{\alpha i j}=\frac{1}{2} \bar{\theta} \Gamma_{M N} \partial_{\alpha} \theta \delta_{[i}^{M} \delta_{j]}^{N}=\bar{\theta} \Gamma_{i j} \partial_{\alpha} \theta, \quad c_{\alpha \beta \gamma}=c_{i j k}=0 .
$$

Although there is another nonzero term $c_{\alpha \beta i}$, it does not contribute to the action. This can be anticipated from the observation that $\widetilde{c}^{i j}$ are the only nonvanishing components of

\footnotetext{
${ }^{3}$ In reduction, we take $g_{M N}\left(X^{M}\right)=g_{m n}\left(X^{m}\right) \oplus g_{i j}\left(X^{i}\right)$ so that $g_{m i}=\partial_{m} g_{i j}=\partial_{i} g_{m n}=0$. That is, $e_{\alpha}^{\widehat{\imath}}=e_{i}^{\widehat{m}}=\partial_{\alpha} e_{i}^{\widehat{\imath}}=\partial_{i} e_{m}^{\widehat{r}}=0$ for vielbeins.
} 
$\widetilde{c}^{\mu \nu}=\frac{1}{6} \epsilon^{\mu \nu \rho \sigma \tau \lambda} c_{\rho \sigma \tau} u_{\lambda}$ as well as $H^{i j}$ in $\mathcal{L}_{2}$, and that $H_{\alpha i j} \wedge c_{\alpha \beta i}=0$ in $S_{W Z}$. That is, the only nonvanishing component of $\mathcal{H}_{\mu \nu \rho}$ and $\widetilde{\mathcal{H}}^{\mu \nu}$ are, respectively,

$$
\mathcal{H}_{\alpha i j}=H_{\alpha i j}-c_{\alpha i j}, \quad \widetilde{\mathcal{H}}^{i j}=\widetilde{H}^{i j}-\widetilde{c}^{i j} .
$$

Finally, $c_{6}$ is evaluated in the similar manner as

$$
c_{6}=-\epsilon^{\alpha \beta}\left(\bar{\theta} \Gamma_{(5)} \Gamma_{m} \partial_{\alpha} \theta \partial_{\beta} X^{m}+\frac{1}{2} \bar{\theta} \Gamma_{(5)} \Gamma_{m} \partial_{\alpha} \theta \bar{\theta} \Gamma^{m} \partial_{\beta} \theta\right)
$$

where $\Gamma_{(5)}=-\Gamma_{78910}$.

Now, note that the nonvanishing components of the above pullbacks $G_{\mu \nu}, c_{3}$, and $c_{6}$, include three kinds of $\theta$ bilinears:

$$
\bar{\theta} \Gamma_{m} \partial_{\alpha} \theta\left(\text { in } c_{6}\right), \quad \bar{\theta} \Gamma_{i} \partial_{\alpha} \theta\left(\text { in } G_{\mu \nu}\right), \quad \bar{\theta} \Gamma_{i j} \partial_{\alpha} \theta\left(=c_{3}\right)
$$

$\left(\bar{\theta} \Gamma_{(5)} \Gamma_{m} \partial_{\alpha} \theta\right.$ in $c_{6}$ is identified to be the same as $\bar{\theta} \Gamma_{m} \partial_{\alpha} \theta$ as shown below.) To complete the reduction of the pullbacks, we need to identify these bilinears using the decomposition of $\theta$ in eq. (3.19) and the properties of the covariantly constant SMW spinors $\chi_{A}$ on K3. The gamma matrix decompositions introduced in eq. (3.21) are used here.

First, let us evaluate $\bar{\theta} \Gamma_{m} \partial_{\alpha} \theta$ in $c_{6}$ :

$$
\begin{aligned}
\bar{\theta} \Gamma_{m} \partial_{\alpha} \theta & =\sum_{A, B}\left(\bar{\lambda}^{A} \otimes \bar{\chi}^{A}\right)\left(\widetilde{\Gamma}_{m} \otimes \widetilde{\Gamma}_{(5)}\right)\left(\partial_{\alpha} \lambda_{B} \otimes \chi_{B}\right) \\
& =\sum_{A, B} \bar{\lambda}^{A} \widetilde{\Gamma}_{m} \partial_{\alpha} \lambda_{B} \bar{\chi}^{A} \chi_{B}
\end{aligned}
$$

where we have used $\bar{\chi}^{1} \widetilde{\Gamma}_{(5)}=\bar{\chi}^{1}$ and $\bar{\chi}^{2} \widetilde{\Gamma}_{(5)}=\bar{\chi}^{2}, \chi_{1}$ and $\chi_{2}$ being positive chirality spinors. Note that in the last line of eq. (3.34) appear the bilinear scalars $\bar{\chi}^{A} \chi_{B}$ on K3. Reminding that $\chi_{1}$ and $\chi_{2}$ are respectively the lowest and the highest (2nd excited) spin states and $\bar{\chi}^{A}=\left(\chi_{A}\right)^{\dagger}$, we can identify those bilinears on K3: $\bar{\chi}^{1} \chi_{2}=\bar{\chi}^{2} \chi_{1}=0$ by the orthogonality between the states; and $\bar{\chi}^{1} \chi_{1}=\bar{\chi}^{2} \chi_{2}=1$ by the constancy of $\chi_{A}$. Then, eq. (3.34) becomes

$$
\bar{\theta} \Gamma_{m} \partial_{\alpha} \theta=\bar{\lambda}^{A} \widetilde{\Gamma}_{m} \partial_{\alpha} \lambda_{A}
$$

By using this result we can complete the reduction of $c_{6}$ which is the first term of $S_{W Z}$. However, note that $\bar{\theta} \Gamma_{(5)} \Gamma_{m} \partial_{\alpha} \theta=\bar{\theta} \Gamma_{m} \partial_{\alpha} \theta$ as can be seen in eq. (3.34), $\chi_{A}$ being of positive chirality, so that in eq. (3.32)

$$
\epsilon^{\alpha \beta} \bar{\theta} \Gamma_{(5)} \Gamma_{m} \partial_{\alpha} \theta \bar{\theta} \Gamma^{m} \partial_{\beta} \theta=\epsilon^{\alpha \beta} \bar{\theta} \Gamma_{m} \partial_{\alpha} \theta \bar{\theta} \Gamma^{m} \partial_{\beta} \theta=0
$$

As a result, inserting eq. (3.35) into eq. (3.32) and integrating over K3,

$$
\int c_{6}=-\epsilon^{\alpha \beta}\left(\bar{\lambda}^{A} \widetilde{\Gamma}_{m} \partial_{\alpha} \lambda_{A}\right) \partial_{\beta} X^{m}
$$


Next, let us evaluate $\bar{\theta} \Gamma_{i} \partial_{\alpha} \theta$ which appears in $G_{\alpha \beta}$ and in $G_{\alpha i}$ :

$$
\begin{aligned}
\bar{\theta} \Gamma_{i} \partial_{\alpha} \theta & =\sum_{A, B}\left(\bar{\lambda}^{A} \otimes \bar{\chi}^{A}\right)\left(1 \otimes \widetilde{\Gamma}_{i}\right)\left(\partial_{\alpha} \lambda_{B} \otimes \chi_{B}\right) \\
& =\sum_{A, B} \bar{\lambda}^{A} \partial_{\alpha} \lambda_{B} \bar{\chi}^{A} \widetilde{\Gamma}_{i} \chi_{B} .
\end{aligned}
$$

Here, the 4-d bilinears $\bar{\chi}^{A} \widetilde{\Gamma}_{i} \chi_{B}$ in the last line are the one-forms on K3. Similarly to the above bilinear scalars, we can identify these bilinears by using the spin sate analysis. But here we should consider the gamma matrices in the bilinears, which are the ladder operators acting on the spin states. For example, $\bar{\chi}^{1} \widetilde{\Gamma}_{\bar{a}} \chi_{1}=0$, because $\widetilde{\Gamma}_{\bar{a}} \chi_{1}$ is the first excited state which is orthogonal to the lowest state $\chi_{1}$. Similarly, $\bar{\chi}^{1} \widetilde{\Gamma}_{a} \chi_{2}=0$ where $\widetilde{\Gamma}_{a} \chi_{2}$ is also the first excited state 4 . Note, besides orthogonality, $\Gamma_{a} \chi_{1}=\Gamma_{\bar{a}} \chi_{2}=0$ gives vanishing bilinears. Then, examining the bilinear one-forms above in this way, we find that all of them vanish, which means,

$$
\bar{\theta} \Gamma_{i} \partial_{\alpha} \theta=0
$$

This makes $G_{\alpha \beta}$ and $G_{\alpha i}$ of eq. (3.29) become

$$
G_{\alpha \beta}=\widetilde{G}_{\alpha \beta}, \quad G_{\alpha i}=0
$$

That is, $G_{\mu \nu}$ becomes block diagonal as in the bosonic case:

$$
G_{\mu \nu}=\widetilde{G}_{\alpha \beta} \oplus h_{i j}, \quad G=\widetilde{G} h
$$

which implies that we get the same reduced expressions as in the bosonic case for $G$ and for the terms contracted by $G_{\mu \nu}$.

Finally, let us evaluate $c_{\alpha i j}=\bar{\theta} \Gamma_{i j} \partial_{\alpha} \theta$, which will be verified to play a key role in the reduction. It becomes

$$
\begin{aligned}
\bar{\theta} \Gamma_{i j} \partial_{\alpha} \theta & =\sum_{A, B}\left(\bar{\lambda}^{A} \otimes \bar{\chi}^{A}\right)\left(1 \otimes \widetilde{\Gamma}_{i j}\right)\left(\partial_{\alpha} \lambda_{A} \otimes \chi_{A}\right) \\
& =\sum_{A, B} \bar{\lambda}^{A} \partial_{\alpha} \lambda_{B} \bar{\chi}^{A} \widetilde{\Gamma}_{i j} \chi_{B} .
\end{aligned}
$$

In this case, the bilinears $\bar{\chi}^{A} \widetilde{\Gamma}_{i j} \chi_{B}$ are the two-forms on K3. As in the previous cases, we can easily check which (components) of these two-forms vanish, by considering the successive ladder operators $\widetilde{\Gamma}_{a}\left(\widetilde{\Gamma}_{\bar{a}}\right)$ between the spin states. For example, $\bar{\chi}^{1} \widetilde{\Gamma}_{\bar{a} \bar{b}} \chi_{1}$, the antiholomorphic components of $\bar{\chi}^{1} \widetilde{\Gamma}_{i j} \chi_{1}$, vanish:

$$
\bar{\chi}^{1} \widetilde{\Gamma}_{\bar{a} \bar{b}} \chi_{1} \sim \bar{\chi}^{1}\left(\widetilde{\Gamma}_{\bar{a}} \widetilde{\Gamma}_{\bar{b}}-\widetilde{\Gamma}_{\bar{b}} \widetilde{\Gamma}_{\bar{a}}\right) \chi_{1} \sim \bar{\chi}^{1} \chi_{2}=0
$$

\footnotetext{
${ }^{4}$ To avoid confusion, we denote the complex coordinates of K3 by $\omega^{a}$ and $\omega^{\bar{a}}$, while the real coordinates are denoted by $\sigma^{i}$.
} 
where the lowest state $\chi_{1}$ has been raised by the two successive raising operators $\widetilde{\Gamma}_{\bar{a}}$ and $\widetilde{\Gamma}_{\bar{b}}$ to be proportional to the highest state $\chi_{2}$. In this way, we can find the vanishing components of each bilinear two-form and can write down the two-forms with the nonvanishing components only. The result is

$$
\begin{aligned}
& \bar{\chi}^{1} \widetilde{\Gamma}_{i j} \chi_{1} d \sigma^{i} \wedge d \sigma^{j}=\bar{\chi}^{1} \widetilde{\Gamma}_{a \bar{b}} \chi_{1} d \omega^{a} \wedge d \omega^{\bar{b}}, \\
& \bar{\chi}^{2} \widetilde{\Gamma}_{i j} \chi_{2} d \sigma^{i} \wedge d \sigma^{j}=\bar{\chi}^{2} \widetilde{\Gamma}_{a \bar{b}} \chi_{2} d \omega^{a} \wedge d \omega^{\bar{b}}, \\
& \bar{\chi}^{1} \widetilde{\Gamma}_{i j} \chi_{2} d \sigma^{i} \wedge d \sigma^{j}=\bar{\chi}^{1} \widetilde{\Gamma}_{a b} \chi_{2} d \omega^{a} \wedge d \omega^{b}, \\
& \bar{\chi}^{2} \widetilde{\Gamma}_{i j} \chi_{1} d \sigma^{i} \wedge d \sigma^{j}=\bar{\chi}^{2} \widetilde{\Gamma}_{\bar{a} \bar{b}} \chi_{1} d \omega^{\bar{a}} \wedge d \omega^{\bar{b}} .
\end{aligned}
$$

Now, let us examine what these two-forms, which constitute $c_{\alpha i j}$, are on K3 and which role they play in the reduction of $\mathcal{H}_{\alpha i j}=H_{\alpha i j}-c_{\alpha i j}$ and of $H_{\alpha i j} \wedge c_{\alpha i j}$.

First, we can show that $\bar{\chi}^{1} \widetilde{\Gamma}_{a \bar{b}} \chi_{1} d \omega^{a} \wedge d \omega^{\bar{b}}$ and $\bar{\chi}^{2} \widetilde{\Gamma}_{a \bar{b}} \chi_{2} d \omega^{a} \wedge d \omega^{\bar{b}}$ in eq. (3.44) are proportional to the Kähler form $J=i g_{a \bar{b}} d \omega^{a} \wedge d \omega^{\bar{b}}$. This is realized as

$$
\begin{aligned}
& \bar{\chi}^{1} \widetilde{\Gamma}_{a \bar{b}} \chi_{1}=\bar{\chi}^{1} g_{a \bar{b}} \chi_{1}-\bar{\chi}^{1} \widetilde{\Gamma}_{\bar{b}} \widetilde{\Gamma}_{a} \chi_{1}=g_{a \bar{b}}, \\
& \bar{\chi}^{2} \widetilde{\Gamma}_{a \bar{b}} \chi_{2}=-\bar{\chi}^{2} g_{a \bar{b}} \chi_{2}+\bar{\chi}^{2} \widetilde{\Gamma}_{a} \widetilde{\Gamma}_{\bar{b}} \chi_{2}=-g_{a \bar{b}},
\end{aligned}
$$

where we have used $\widetilde{\Gamma}_{a} \chi_{1}=\widetilde{\Gamma}_{\bar{b}} \chi_{2}=0$ and normalization $\bar{\chi}^{1} \chi_{1}=\bar{\chi}^{2} \chi_{2}=1, \chi_{1}$ and $\chi_{2}$ being covariantly constant. One property of the Kähler form $J$ is that it is self-dual and harmonic. It is self-dual because, up to a positive constant factor,

$$
* g_{a \bar{b}} d \omega^{a} \wedge d \omega^{\bar{b}} \sim-\sqrt{g} g_{a \bar{b}} \epsilon_{\bar{c}}^{a} \epsilon_{d}^{\bar{b}} d \omega^{\bar{c}} \wedge d \omega^{d} \sim g_{d \bar{c}} d \omega^{d} \wedge d \omega^{\bar{c}}
$$

where the Hermitian property of the metric, $g_{a b}=g_{\bar{a} \bar{b}}=0$ has been used. Then since Kähler form $J$ is closed and is self-dual as shown in above, it is coclosed as well. Therefore, it is harmonic.

One can show $\Omega=\bar{\chi}^{1} \widetilde{\Gamma}_{a b} \chi_{2} d \omega^{a} \wedge d \omega^{b}$ and $\bar{\Omega}=\bar{\chi}^{2} \widetilde{\Gamma}_{\bar{a} \bar{b}} \chi_{1} d \omega^{\bar{a}} \wedge d \omega^{\bar{b}}$ in eq. (3.44) are also self-dual and harmonic by a similar manner. Thus we have identified the nonvanishing bilinears in eq. (3.44) as three independent self-dual harmonic two-forms $J, \Omega$ and $\bar{\Omega}$ on K3. These three self-dual two-forms can be transformed into the real ones, $J, \frac{1}{2}(\Omega+\bar{\Omega})$ and $\frac{1}{2 i}(\Omega-\bar{\Omega})$, which are the self-dual elements of $H^{2}(\mathrm{~K} 3, \mathbb{R})$.

Then, the point is that we can take the harmonic two-form basis $b_{I i j}$ on K3, consisting of 19 anti-self-dual forms and 3 self-dual forms, such that the above three real harmonic two-forms are the self-dual elements of the basis: 5 :

$$
b_{1}^{+}=J, \quad b_{2}^{+}=\frac{1}{2}(\Omega+\bar{\Omega}), \quad b_{3}^{+}=\frac{1}{2 i}(\Omega-\bar{\Omega}) .
$$

As a result of the above identification of the bilinear two-forms in eq. (3.44), we can rewrite $c_{\alpha i j}=\bar{\theta} \Gamma_{i j} \partial_{\alpha} \theta$ of eq. (3.42) as

$$
c_{\alpha i j}=\sum_{I=1}^{3} c_{\alpha}^{I} b_{I i j}^{+}
$$

\footnotetext{
${ }^{5}$ We denote the anti-self-dual and the self-dual harmonic two-forms by $b_{I}^{-}$and $b_{I}^{+}$, respectively.
} 
where we have rearranged the 7 -d bilinears $\bar{\lambda}^{A} \partial_{\alpha} \lambda_{B}$ in eq. (3.42) to be the coefficients $c_{\alpha}^{I}$ of the self-dual basis $b_{I}^{+}$:

$$
\begin{aligned}
& c_{\alpha}^{1}=-i\left(\bar{\lambda}^{1} \partial_{\alpha} \lambda_{1}-\bar{\lambda}^{2} \partial_{\alpha} \lambda_{2}\right), \\
& c_{\alpha}^{2}=\bar{\lambda}^{1} \partial_{\alpha} \lambda_{2}+\bar{\lambda}^{2} \partial_{\alpha} \lambda_{1}, \\
& c_{\alpha}^{3}=i\left(\bar{\lambda}^{1} \partial_{\alpha} \lambda_{2}-\bar{\lambda}^{2} \partial_{\alpha} \lambda_{1}\right) .
\end{aligned}
$$

That is, $c_{\alpha i j}$ is expanded into only the self-dual part of the harmonic basis $H^{2}(\mathrm{~K} 3, \mathbb{R})$, whereas $H_{\alpha i j}$ is expanded into the whole basis as in eq. (2.6). As a result, $\mathcal{H}_{\alpha i j}=H_{\alpha i j}-c_{\alpha i j}$, the only contributing components of $\mathcal{H}_{\mu \nu \rho}$, can be expanded as

$$
\mathcal{H}_{\alpha i j}=\sum_{I=1}^{19} \partial_{\alpha} Y^{I} b_{I i j}^{-}+\sum_{I=1}^{3} Z_{\alpha}^{I} b_{I i j}^{+},
$$

where the coefficients of the self-dual part are the supersymmetric quantities,

$$
Z_{\alpha}^{I}=\partial_{\alpha} Y^{I}-c_{\alpha}^{I}
$$

Then, $\widetilde{\mathcal{H}}^{i j}$, the only nonvanishing components of $\widetilde{\mathcal{H}}^{\mu \nu}$ become

$$
\widetilde{\mathcal{H}}^{i j}=\sqrt{h}\left(\sum_{I=1}^{19} \widetilde{Y}^{I}\left(* b_{I}^{-}\right)^{i j}+\sum_{I=1}^{3} \widetilde{Z}^{I}\left(* b_{I}^{+}\right)^{i j}\right),
$$

where $\widetilde{Z}^{I}=\epsilon^{\alpha \beta} Z_{\alpha}^{I} u_{\beta}$.

We can interpret this result in terms of the dual description of the heterotic string as in the bosonic case. In fact, the self-duality of $H_{\alpha i j}$ forces it to be expanded as

$$
H_{\alpha i j}=\sum_{I=1}^{19} \partial_{\alpha} Y_{-}^{I} b_{I i j}^{-}+\sum_{I=1}^{3} \partial_{\alpha} Y_{+}^{I} b_{I i j}^{+}
$$

where $Y_{-}^{I}$ and $Y_{+}^{I}$ corresponds to the left-movers and the right-movers of the heterotic string, respectively. (We will use terms left movers and right movers for $Y_{-}^{I}$ and $Y_{+}^{I}$ but this terminology is more appropriate only after the gauge fixing. The situation is the same for the self-duality of $H_{\alpha i j}$, which is manifest after gauge fixing.) Then, when the heterotic string is compactified on $T^{3}$, the left movers $Y_{-}^{I}$ which is bosonic in 10-d should remain bosonic, whereas the right-movers $Y_{+}^{I}$ should remain supersymmetric as in 10-d. This is well satisfied by eq. (3.50), where $c_{\alpha}^{I}$, the fermionic bilinears in 7-d, are added only to the three right-movers $Y_{+}^{I}$ making $Z_{\alpha}^{I}$ supersymmetric.

Now if we insert $\mathcal{H}_{\alpha i j}$ and $\widetilde{\mathcal{H}}^{i j}$ into $\mathcal{L}_{1}$ and $\mathcal{L}_{2}$ of the supersymmetric action, as in the bosonic case, the related terms are written in terms of $\int b_{I} \wedge b_{J}$ and $\int b_{I} \wedge * b_{J}$. However, note

$$
\int b_{I}^{-} \wedge b_{J}^{+}=\int b_{I}^{-} \wedge * b_{J}^{+}=\int b_{J}^{+} \wedge * b_{I}^{-}=\int-b_{I}^{-} \wedge b_{J}^{+}=0 .
$$


That is, the product of a anti-self-dual two-form and a self-dual two-form vanishes. As a result, as $\mathcal{L}_{1}$ and $\mathcal{L}_{2}$ are integrated over K3, $L_{I J}=\int_{K 3} b_{I} \wedge b_{J}$ and $M_{I J}=\int_{K 3} b_{I} \wedge * b_{J}$ of eq. (2.8) become block diagonal:

$$
L=L^{-} \oplus L^{+}, \quad M=M^{-} \oplus M^{+}
$$

where $L^{-}\left(M^{-}\right)$and $L^{+}\left(M^{+}\right)$are the blocks constructed from the anti-self-dual and the self-dual elements of the harmonic basis, respectively: $L_{I J}^{-}=\int_{K 3} b_{I}^{-} \wedge b_{J}^{-}, L_{I J}^{+}=\int_{K 3} b_{I}^{+} \wedge b_{J}^{+}$; and similarly for $M_{I J}^{-}, M_{I J}^{+}$.

The above decomposition of $L$ and $M$ makes all terms including $L$ and $M$ decomposed into the terms including the coefficients of the self-dual forms and those of the anti-self-dual forms. This implies that the left-movers and the right-movers in eq. (3.50) are decoupled in the action as expected for the dual heterotic string action. For example, $\mathcal{L}_{2}$ becomes

$$
\begin{aligned}
\int_{K 3} \widetilde{\mathcal{H}}^{i j} \mathcal{H}_{\alpha i j} u^{\alpha} & \sim \int_{K 3} \sqrt{h}\left(\widetilde{Y}^{I} *\left(* b_{I}^{-} \wedge * b_{J}^{-}\right) \partial_{\alpha} Y^{J}+\widetilde{Z}^{I} *\left(* b_{I}^{+} \wedge * b_{J}^{+}\right) Z_{\alpha}^{J}\right) \\
& \sim\left(\widetilde{Y}^{I} L_{I J}^{-} \partial_{\alpha} Y^{J}+\widetilde{Z}^{I} L_{I J}^{+} Z_{\alpha}^{J}\right) u^{\alpha}
\end{aligned}
$$

where we can see the terms of left-movers, $\widetilde{Y}^{I} L_{I J}^{-} \partial_{\alpha} Y^{J}$ and those of right-movers, $\widetilde{Z}^{I} L_{I J}^{+} Z_{\alpha}^{J}$ are separated.

In this way, inserting $G_{\mu \nu}$ of eq. (3.41) besides $\mathcal{H}_{\alpha i j}$ and $\widetilde{\mathcal{H}}^{i j}$ into $\mathcal{L}_{1}$ and $\mathcal{L}_{2}$, and integrating over $\mathrm{K} 3$,

$$
\begin{aligned}
& \mathcal{L}_{1} \rightarrow-\sqrt{-G} \sqrt{1+\frac{\widetilde{Y} M^{-} \widetilde{Y}+\widetilde{Z} M^{+} \widetilde{Z}}{G u^{2}}+\left(\frac{\widetilde{Y} L^{-} \widetilde{Y}+\widetilde{Z} L^{+} \widetilde{Z}}{2 G u^{2}}\right)^{2}}, \\
& \mathcal{L}_{2} \rightarrow-\frac{\left(\widetilde{Y} L^{-} \partial_{\alpha} Y+\widetilde{Z} L^{+} Z_{\alpha}\right) u^{\alpha}}{2 u^{2}}
\end{aligned}
$$

where we have omitted the basis indices $I, J$.

Thus we have completed the reduction of $\mathcal{L}_{1}$ and $\mathcal{L}_{2}$. Besides, we have already reduced the first term of $S_{W Z}$ in eq. (3.37). Then the rest part is the second term of $S_{W Z}$, which is $\frac{1}{2} H \wedge c_{3}$. This term is easily reduced by using $c_{\alpha i j}$ in eq. (3.48) and $H_{\alpha i j}$ in eq. (2.6):

$$
\begin{aligned}
\frac{1}{2} \int_{K 3} H \wedge c_{3} & =\frac{1}{4} \int_{K 3} \epsilon^{\alpha \beta} \epsilon^{i j k l} H_{\alpha i j} c_{\beta i j} \\
& =\int_{K 3} \epsilon^{\alpha \beta} \sqrt{h} \partial_{\alpha} Y^{I}\left(b_{I}^{+} \wedge b_{J}^{+}\right) c_{\beta}^{J} \\
& =\epsilon^{\alpha \beta} \partial_{\alpha} Y L^{+} c_{\beta} .
\end{aligned}
$$

Note that this term has only the block of the self-dual basis.

Finally, collecting the results of the reductions, eq. (3.37), (3.57), and (3.58), the full 
action of the 7 -d heterotic string becomes

$$
\begin{aligned}
\mathcal{L}_{1} & =-\sqrt{-G} \sqrt{1+\frac{\widetilde{Y} M^{-} \widetilde{Y}+\widetilde{Z} M^{+} \widetilde{Z}}{G u^{2}}+\left(\frac{\widetilde{Y} L^{-} \widetilde{Y}+\widetilde{Z} L^{+} \widetilde{Z}}{2 G u^{2}}\right)^{2}}, \\
\mathcal{L}_{2} & =-\frac{\left(\widetilde{Y} L^{-} \partial_{\alpha} Y+\widetilde{Z} L^{+} Z_{\alpha}\right) u^{\alpha}}{2 u^{2}} \\
\mathcal{L}_{3} & =-\epsilon^{\alpha \beta}\left(\bar{\lambda}^{A} \widetilde{\Gamma}_{m} \partial_{\alpha} \lambda_{A}\right) \partial_{\beta} X^{m} \\
\mathcal{L}_{4} & =-\epsilon^{\alpha \beta}\left(\partial_{\alpha} Y L^{+} c_{\beta}\right) .
\end{aligned}
$$

\section{10-d Heterotic String Action and Its Kappa Symmetry}

We now try to construct the 10-d action of the heterotic string by using the structure of the 7 - $\mathrm{d}$ action eq. (3.59). The 10-d action can be naturally constructed by considering the intersection matrix $L$ above along with the left-moving and the right-moving part of the 7-d action.

As mentioned in the bosonic reduction, the topology of $K 3$ requires $L=\int_{K 3} b_{I} \wedge b_{J}$ to be the intersection matrix of the even self-dual lattice with signature $(19,3)$. This lattice is unique up to isometries which preserve the inner product, so that one can choose $L$ as [15]

$$
L=-E_{8} \oplus-E_{8} \oplus \sigma \oplus \sigma \oplus \sigma
$$

where $E_{8}$ is the Cartan matrix of the Lie group $E_{8}$, and $\sigma=\left(\begin{array}{ll}0 & 1 \\ 1 & 0\end{array}\right)$.

In the case of 10-d heterotic string, the 16 modes of the left-movers construct an even self-dual lattice of signature $(16,0)$. There are two such lattices, one of which is $\Gamma_{8} \times \Gamma_{8}$ with $\Gamma_{8}$ being the root lattice of $E_{8}$. Then, as we construct the 10-d heterotic action from the 7 - $\mathrm{d}$ action, it is natural to eliminate the block $\Sigma=\sigma \oplus \sigma \oplus \sigma$ of signature $(3,3)$ in eq. (4.1) along with the elements in the action that couple to $\Sigma$.

In fact, $L^{+}$, the intersection matrix of the three self-dual two-forms, $J, \frac{1}{2}(\Omega+\bar{\Omega})$ and $\frac{1}{2 i}(\Omega-\bar{\Omega})$, has the signature of $(0,3)$. Therefore, $L^{+}$forms the sub-block of $\Sigma$ whose signature is $(3,3)$, so that we can eliminate $L^{+}$along with the $Z_{\alpha}^{I}$ and $\widetilde{Z}^{I}$ which couple to it. Since $Z_{\alpha}^{I}=\partial_{\alpha} Y^{I}-c_{\alpha}^{I}$ is the supersymmetric extension of right-movers $\partial_{\alpha} Y_{+}^{I}$, the elimination of these terms naturally give the 10 -d theory and we are left with scalar degrees of freedom representing $E_{8} \times E_{8}$ current algebra.

Consequently, keeping only the terms including the sub-block $L=-E_{8} \oplus-E_{8}$ of $L^{-}$ 
(and similarly for $M^{-}$), its signature being $(16,0)$, the proposed 10-d action is written as

$$
\begin{aligned}
\mathcal{L}_{1} & =-\sqrt{-G} \sqrt{1+\frac{\widetilde{Y} M \widetilde{Y}}{G u^{2}}+\left(\frac{\tilde{Y} L \tilde{Y}}{2 G u^{2}}\right)^{2}}, \\
\mathcal{L}_{2} & =-\frac{\tilde{Y} L \partial_{\alpha} Y u^{\alpha}}{2 u^{2}}, \\
\mathcal{L}_{3} & =-\epsilon^{\alpha \beta} \bar{\theta} \Gamma_{m} \partial_{\alpha} \theta \partial_{\beta} X^{m} \quad(m=1, \cdots, 10), \\
\mathcal{L}_{4} & =0 .
\end{aligned}
$$

Here, $\mathcal{L}_{4}=0$ because it has contained only $L^{+}$terms in 7 -d. Also note that we have added three noncompact coordinates $X^{m}$ which enter the induced metric $G_{\alpha \beta}$ in $\mathcal{L}_{1}$ as well as in $\mathcal{L}_{3}$. The above 10-d action has correct field contents of the heterotic string. $Y^{I}$ are the 16 scalars and $X^{m}$ are coordinates of 10 dimensions while $\theta$ is a Majorana-Weyl spinor of 10-d with 16 real degrees of freedom.

Note that in the above 10-d action the condition $M L^{-1} M=L$ in $(10-n)$ dimension [1] reduces to $M=-L$. This is realized from the M5-brane point of view as $L_{I J}=\int_{K 3} b_{I} \wedge b_{J}$ and $M_{I J}=\int_{K 3} b_{I} \wedge * b_{J}$ where $b_{I}$ are taken to be all anti-self-dual when they construct $(16,0)$ block, as argued above.

The condition $M=-L$ is also well understood in terms of the 10-d heterotic string. This can be seen first from the moduli space of the theory. In $(10-n)$ dimension matrix $M$ characterizes the Narain moduli space, up to the T-duality group $O(16+n, n ; \mathbb{Z})[1$,

$$
O(16+n, n) /(O(16+n) \times O(n))
$$

Then, in 10-d $(n=0)$ the moduli space M characterizes becomes a 0-dimensional space so that $M$ is a matrix of fixed components for corresponding $L$. The concrete form $M=-L$ can be justified by the projections $\mathcal{P}_{ \pm}=\frac{1}{2}\left(1 \pm L^{-1} M\right)$, for $\left(L^{-1} M\right)^{2}=1$, which projects out right- and left-moving $Y^{I}$. Then, in $10-\mathrm{d} \mathcal{P}_{+}=0$ is needed, since there are no right movers in compact dimension. As a result, we have $M=-L$.

The equation of motion of $Y^{I}$ in terms of $P_{-}$along with the identification of $Y^{I}$ as chiral bosons (left-movers) in 10-d action can be given by the PST gauge as follows. First we can obtain the compact part of the 10-d action in Polyakov type in a similar way to the 7-d bosonic string described in ref. [1]:

$$
\mathcal{L}_{C}=-\frac{1}{2 u^{2}} \tilde{Y} L(\widetilde{Y}+\partial Y \cdot u)
$$

where $M=-L$ has been used and $u_{\alpha}=\partial_{\alpha} a$. This compact part (as well as the full action) is invariant under the two kinds of PST gauge transformations

$$
\begin{aligned}
& \delta Y=\phi \frac{\mathcal{P}_{-} \partial_{+} Y}{\partial_{+} a}, \quad \delta a=\phi, \\
& \delta Y=f(a), \quad \delta a=0,
\end{aligned}
$$


where the lattice indices $I$ of $Y^{I}$ are suppressed. Then fixing the second gauge in eq. (4.5), similarly to the cases introduced in ref. [21], we can identify the $Y^{I}$ in the action as leftmovers. As we write down the action in eq. (4.4) as

$$
\mathcal{L}_{C}=-\frac{1}{2 u^{2}} u^{\alpha} F_{\alpha}^{*} L\left(F_{\beta}^{*}+F_{\beta}\right) u^{\beta},
$$

where $F_{\alpha}=\partial_{\alpha} Y, F_{\alpha}^{*}=\epsilon_{\alpha}^{\beta} \partial_{\beta} Y$, the e.o.m. for $Y^{I}$ becomes

$$
\epsilon^{\alpha \beta} \partial_{\alpha}\left(\frac{u_{\beta} \mathcal{F}_{\gamma} u^{\gamma}}{u^{2}}\right)=\epsilon^{\alpha \beta} \partial_{\alpha}\left(\frac{\mathcal{F}}{u_{+}}\right) u_{\beta}=0,
$$

where $\mathcal{F}=\mathcal{F}_{\alpha}=F_{\alpha}^{*}+F_{\alpha}=F_{0}+F_{1}=\partial_{+} Y$. The general solution of the e.o.m. (4.7) is

$$
\mathcal{F}=g(a) u_{+}
$$

where $g(a)$ is an arbitrary scalar function of $a$. However, we find that this solution is equal to the pure gauge $\delta \mathcal{F}$ for the 2nd gauge symmetry of (4.5):

$$
\delta \mathcal{F}=\partial_{+} \delta Y=\partial_{+} f(a)=g(a) u_{+}
$$

where $g(a)=f^{\prime}(a)$. Therefore, we can pick up the solution

$$
\mathcal{F}=F_{\alpha}^{*}+F_{\alpha}=\mathcal{P}_{-} \partial_{+} Y=0,
$$

as a gauge fixed e.o.m., $F_{\alpha}$ being anti-self-dual and $Y^{I}$ left-movers. (For $L=M$, we get the self-dual $F_{\alpha}$ and right moving $Y^{I}$.)

Another clue for $M=-L$ is the kappa invariance of the $10 \mathrm{~d}$ action, which is not achieved unless $M=-L$ as will be shown below and in the appendix. Thus the condition $M=-L$ originated from the M5-brane side is consistent with 10 -d heterotic string. We emphasize that at the classical level we don't have to impose $L$ to be intersection matrix of the even self-dual lattice with signature $(16,0)$. Such condition would arise from the absence of the world-sheet anomaly, which would arise at one-loop. It would be interesting to work out this explicitly.

Now, let us check the kappa symmetry of the above 10-d action to see it is the correct one. We can infer, from the kappa variation of the fields of M5-brane action, the kappa variations of the $10-\mathrm{d}$ fields

$$
\delta \bar{\theta}=\bar{\kappa}(1-\Gamma), \quad \delta X^{m}=-\delta \bar{\theta} \Gamma^{m} \theta, \quad \delta Y^{I}=0 .
$$

Note $\delta Y^{I}=0$ which is satisfied in the absence of the Yang-Mills background $A_{m}^{I}$. That is, the kappa variations of 16 left-movers in compact dimensions vanish. Therefore, we can consider the variations of $X^{m}$ and $\theta$ of noncompact 10-d only.

From above variations of $X^{m}$ and $\theta$ we can deduce the variation of the pullback vielbein $\Pi_{\alpha}^{\widehat{m}}$. Since here we take the $10-\mathrm{d}$ supergravity background to be flat,

$$
\Pi_{\alpha}^{\widehat{m}}=\Pi_{\alpha}^{m}=\partial_{\alpha} X^{m}-\bar{\theta} \Gamma^{m} \partial_{\alpha} \theta,
$$


whose kappa variation becomes

$$
\delta \Pi_{\alpha}^{m}=-2 \delta \bar{\theta} \Gamma^{m} \partial_{\alpha} \theta
$$

Then, we get the variations of induced metric $G_{\alpha \beta}$ and the related quantities as

$$
\begin{aligned}
\delta G_{\alpha \beta} & =-2 \delta \bar{\theta} \gamma_{\{\alpha} \partial_{\beta\}} \theta, \\
\delta G^{\alpha \beta} & =-G^{\alpha \gamma} \delta G_{\gamma \delta} G^{\delta \beta}=2 \delta \bar{\theta} \gamma^{\{\alpha} \partial^{\beta\}} \theta, \\
\delta G & =\frac{1}{2} \epsilon^{\alpha \beta} \epsilon^{\gamma \delta} \delta\left(G_{\alpha \gamma} G_{\beta \delta}\right)=-4 G \delta \bar{\theta} \gamma^{\alpha} \partial_{\alpha} \theta, \\
\delta u^{2} & =\delta G^{\alpha \beta} u_{\alpha} u_{\beta}=4 \delta \bar{\theta} \gamma^{\alpha} \partial^{\beta} \theta u_{\alpha} u_{\beta},
\end{aligned}
$$

where $\gamma_{\alpha}=\Gamma_{m} \Pi_{\alpha}^{m}$.

We can prove the kappa symmetry of the action by using the variations above. The main scheme is similar to the one introduced in ref. 44 for the case of M5-brane action. First, define $U^{\alpha}$ and $T^{\alpha}$ as

$$
\begin{aligned}
\delta \mathcal{L}_{1} & =\frac{2}{\mathcal{L}_{1}} \delta \bar{\theta} U^{\alpha} \partial_{\alpha} \theta, \\
\delta \mathcal{L}_{2}+\delta \mathcal{L}_{3} & =2 \delta \bar{\theta} T^{\alpha} \partial_{\alpha} \theta .
\end{aligned}
$$

Evaluation of $\delta \mathcal{L}_{1}, \delta \mathcal{L}_{2}$, and $\delta \mathcal{L}_{3}$ gives

$$
\begin{aligned}
U^{\alpha} & =G \gamma^{\alpha}+\frac{\widetilde{Y} M \widetilde{Y}}{\left(u^{2}\right)^{2}} \gamma^{\beta} u_{\beta} u^{\alpha}+\frac{(\widetilde{Y} L \tilde{Y})^{2}}{4 G\left(u^{2}\right)^{3}}\left(2 \gamma^{\beta} u^{\alpha}-\gamma^{\alpha} u^{\beta}\right) u_{\beta}, \\
T^{\alpha} & =-\bar{\gamma} \gamma^{\alpha}+\frac{\tilde{Y} L \tilde{Y}}{2\left(u^{2}\right)^{2}} \epsilon_{\beta \gamma} \gamma^{\{\beta} G^{\delta\} \alpha} u^{\gamma} u_{\delta},
\end{aligned}
$$

where $\bar{\gamma}=\gamma_{01}$. Then, we can take a quantity

$$
\rho=\bar{\gamma}-\frac{\widetilde{Y} L \widetilde{Y}}{2 G u^{2}} \bar{\gamma}
$$

which satisfies, for $M=-L$,

$$
U^{\alpha}=\rho T^{\alpha}, \quad \rho^{2}=\left(\mathcal{L}_{1}\right)^{2} .
$$

Eq. (4.18) above implies that the action is invariant under the kappa symmetry. To see this, first note that we can take $\delta \theta=\bar{\kappa}(1-\Gamma)$ with $\Gamma=\frac{\rho}{\mathcal{L}_{1}}$ because $\Gamma^{2}=1$ from $\rho^{2}=\left(\mathcal{L}_{1}\right)^{2}$. Then, by using $U^{\alpha}=\rho T^{\alpha}$, we find that

$$
\begin{aligned}
\delta \mathcal{L} & =2 \delta \bar{\theta}\left(\frac{U^{\alpha}}{\mathcal{L}_{1}}+T^{\alpha}\right) \partial_{\alpha} \theta \\
& =2 \bar{\kappa}(1-\Gamma)(1+\Gamma) T^{\alpha} \partial_{\alpha} \theta \\
& =0 .
\end{aligned}
$$


The details of the proof, including the evaluation of $U^{\alpha}, T^{\alpha}$, and $\rho$ along with the check of eq. (4.18), are given in the appendix.

The requirement of the kappa invariance restricts the supersymmetry of the heterotic action to be $\mathcal{N}=1$. For example, let us check the possibility of kappa invariance for the case of $\mathcal{N}=2$ supersymmetry such that $\theta=\theta_{1}+\theta_{2}, \theta_{2}$ and $\theta_{2}$ being 16-component Majorana-Weyl spinors. Then, $\mathcal{L}_{1}$ and $\mathcal{L}_{2}$ have the same form as in the eq. (4.2) with $\bar{\theta} \Gamma^{m} \partial_{\alpha} \theta$ replaced by $\bar{\theta}_{A} \Gamma^{m} \partial_{\alpha} \theta_{A}$ for $A=1,2$. But $\mathcal{L}_{3}\left(=\mathcal{L}_{W Z}\right)$ is written as

$$
\mathcal{L}_{3}=-\epsilon^{\alpha \beta}\left(\bar{\theta}_{1} \Gamma_{m} \partial_{\alpha} \theta_{1}-\bar{\theta}_{2} \Gamma_{m} \partial_{\alpha} \theta_{2}\right) \partial_{\beta} X^{m}+\epsilon^{\alpha \beta} \bar{\theta}_{1} \Gamma_{m} \partial_{\alpha} \theta_{1} \bar{\theta}_{2} \Gamma^{m} \partial_{\beta} \theta_{2}
$$

which is the same as $\mathcal{L}_{W Z}$ in Type II theories. Then, under kappa transformations, all terms in $\delta \mathcal{L}_{1}, \delta \mathcal{L}_{2}$ and $\delta \mathcal{L}_{3}$ are written in terms of $\delta \bar{\theta}_{1}$ and $\delta \bar{\theta}_{2}$ separately:

$$
\begin{aligned}
\delta \mathcal{L}_{1} & =\frac{2}{\mathcal{L}_{1}}\left(\delta \bar{\theta}_{1} U^{\alpha} \partial_{\alpha} \theta_{1}+\delta \bar{\theta}_{2} U^{\alpha} \partial_{\alpha} \theta_{2}\right), \\
\delta \mathcal{L}_{2} & =2\left(\delta \bar{\theta}_{1} R^{\alpha} \partial_{\alpha} \theta_{1}+\delta \bar{\theta}_{2} R^{\alpha} \partial_{\alpha} \theta_{2}\right) \\
\delta \mathcal{L}_{3} & =2\left(\delta \bar{\theta}_{1} S^{\alpha} \partial_{\alpha} \theta_{1}-\delta \bar{\theta}_{2} S^{\alpha} \partial_{\alpha} \theta_{2}\right)
\end{aligned}
$$

where $U^{\alpha}$ and $T^{\alpha}=R^{\alpha}+S^{\alpha}\left(S^{\alpha}=-\bar{\gamma} \gamma^{\alpha}\right)$ are those of eq. (4.16) with $\bar{\theta} \Gamma^{m} \partial_{\alpha} \theta$ replaced by $\bar{\theta}_{A} \Gamma^{m} \partial_{\alpha} \theta_{A}$. Here, for $\delta L$ to vanish, $\delta \bar{\theta}_{1}$ and $\delta \bar{\theta}_{2}$ terms should vanish separately. However, this is not possible due to the sign difference between the two terms in $\delta \mathcal{L}_{3}$. That is, referring to eq. (4.18) and (4.19), we need to have for kappa invariance

$$
U^{\alpha}=\rho_{1}\left(R^{\alpha}+S^{\alpha}\right), \quad U^{\alpha}=\rho_{2}\left(R^{\alpha}-S^{\alpha}\right), \quad \rho_{1}^{2}=\rho_{2}^{2}=\left(\mathcal{L}_{1}\right)^{2},
$$

which cannot be satisfied simultaneously. Thus the action is not kappa invariant.

In contrast to the heterotic case, Type II theories have kappa symmetry with $\theta=\theta_{1}+\theta_{2}$. A Type II action in flat background can be given from the above heterotic action by taking $Y^{I}=0$ and $\theta=\theta_{1}+\theta_{2}$ :

$$
\begin{aligned}
& \mathcal{L}_{1}=-\sqrt{-G} \\
& \mathcal{L}_{2}=0 \\
& \mathcal{L}_{3}=-\epsilon^{\alpha \beta}\left(\bar{\theta}_{1} \Gamma_{m} \partial_{\alpha} \theta_{1}-\bar{\theta}_{1} \Gamma_{m} \partial_{\alpha} \theta_{1}\right) \partial_{\beta} X^{m}+\epsilon^{\alpha \beta} \bar{\theta}_{1} \Gamma_{m} \partial_{\alpha} \theta_{1} \bar{\theta}_{2} \Gamma^{m} \partial_{\beta} \theta_{2} .
\end{aligned}
$$

Here $\mathcal{L}_{2}=0$ is critical for the kappa invariance of the action. The action transforms as

$$
\begin{aligned}
& \delta \mathcal{L}_{1}=\frac{2}{\mathcal{L}_{1}}\left(\delta \bar{\theta}_{1} U^{\alpha} \partial_{\alpha} \theta_{1}+\delta \bar{\theta}_{2} U^{\alpha} \partial_{\alpha} \theta_{2}\right), \\
& \delta \mathcal{L}_{3}=2\left(\delta \bar{\theta}_{1} S^{\alpha} \partial_{\alpha} \theta_{1}-\delta \bar{\theta}_{2} S^{\alpha} \partial_{\alpha} \theta_{2}\right)
\end{aligned}
$$

where $U^{\alpha}$ is different from those of eq. (4.21). Then it is possible to take

$$
U^{\alpha}=\rho S^{\alpha}, \quad \rho^{2}=\left(\mathcal{L}_{1}\right)^{2}
$$


which results in, for $\Gamma=\frac{\rho}{L_{1}}$,

$$
\delta \mathcal{L}=2 \delta \bar{\theta}_{1}(1+\Gamma) S^{\alpha} \partial_{\alpha} \theta_{1}+2 \delta \bar{\theta}_{2}(1-\Gamma) S^{\alpha} \partial_{\alpha} \theta_{2}=0
$$

for the $\theta_{A}$ variations

$$
\delta \bar{\theta}_{1}=\bar{\kappa}_{1}(1-\Gamma), \quad \delta \bar{\theta}_{2}=\bar{\kappa}_{2}(1+\Gamma) .
$$

Here the $\delta \theta_{1}$ and $\delta \theta_{2}$ terms vanish separately.

\section{Discussions}

In this paper we propose the supersymmetric heterotic string action motivated by the dimensional reduction of M5-brane wrapping on K3. And we explicitly prove the kappa symmetry of the resulting heterotic action. The novelty is the additional scalars realizing the chiral current algebra. It would be interesting to explicitly verify underlying KacMoody algebras. Also one should consider the generalization of the heterotic action in an arbitrary supergravity background. This construction can also be viewed as D1-D9 system in the Type IIB theory with treating D9s as backgrounds. In Type IIB, there is also D9-D5 system, which is supersymmetric. Its DBI type action is unknown. It would be interesting to work out D9-D5 action and we hope that the current construction can shed some light on that problem.

\section{Acknowledgments}

J.P. is supported in part by the KOSEF SRC Program through CQUeST at Sogang Univer-

sity, by KOSEF Grant R01-2008-000-20370-0 and by the Stanford Institute for Theoretical Physics.

\section{Appendix}

\section{A. Proof of the Kappa Symmetry in 10-d Action}

\section{A.1. Useful relations}

For $\gamma_{\alpha}=\Gamma_{m} \Pi_{\alpha}^{m}$ and $\bar{\gamma}=\gamma_{01}$

$$
\begin{gathered}
\bar{\gamma} \gamma_{\alpha}=-G \epsilon_{\alpha \beta} \gamma^{\beta}, \quad \bar{\gamma} \gamma_{\alpha \beta}=-G \epsilon_{\alpha \beta}, \\
\gamma_{\alpha} \gamma_{\beta}=\gamma_{\alpha \beta}+G_{\alpha \beta}, \quad \gamma_{\alpha \beta} \gamma_{\gamma}=\gamma_{[\alpha} G_{\beta] \gamma} .
\end{gathered}
$$


The generalization to a worldvolume of arbitrary dimension is, for $\bar{\gamma}=\gamma_{1 \cdots D}$,

$$
\begin{aligned}
\bar{\gamma} \gamma_{\mu_{k+1} \cdots \mu_{D}} & =(-)^{\frac{(k+1)(k+2)}{2}} \frac{G}{k !} \epsilon_{\mu_{1} \cdots \mu_{D}} \gamma^{\mu_{1} \cdots \mu_{k}}, \\
\gamma_{\mu_{1} \cdots \mu_{m}} \gamma_{\nu_{1} \cdots \nu_{n}} & =\sum_{k=0}^{\min (m, n)} C_{k}^{m n} \gamma_{\mu_{1} \cdots \mu_{m-k} \nu_{1} \cdots \nu_{n-k}} G_{\mu_{m-k+1} \nu_{n-k+1}} \cdots G_{\mu_{m} \nu_{n}},
\end{aligned}
$$

where $C_{k}^{m n} \equiv(-)^{k n+k(k+1) / 2} k !\left(\begin{array}{c}m \\ k\end{array}\right)\left(\begin{array}{c}n \\ k\end{array}\right)$ and $\mu$ 's and $\nu$ 's in the RHS are antisymmetrized separately.

\section{A.2. Evaluation of $U^{\alpha}$}

First, let us evaluate $\delta\left(\mathcal{L}_{1}\right)^{2}$ to get $U^{\alpha}$ from $\delta\left(\mathcal{L}_{1}\right)^{2}=2 \mathcal{L}_{1} \delta \mathcal{L}_{1}=4 \delta \bar{\theta} U^{\alpha} \partial_{\alpha} \theta$. Since $\delta \widetilde{Y}^{I}=0$ from $\delta Y^{I}=0$, we can consider $\delta G$ and $\delta u^{2}$ above only:

$$
\begin{aligned}
\delta\left(\mathcal{L}_{1}\right)^{2} & =-\delta G-(\widetilde{Y} M \widetilde{Y}) \delta\left(\frac{1}{u^{2}}\right)-\frac{(\widetilde{Y} L \tilde{Y})^{2}}{4} \delta\left(\frac{1}{G\left(u^{2}\right)^{2}}\right) \\
& =4 \delta \bar{\theta}\left(G \gamma^{\alpha}+\frac{\tilde{Y} M \tilde{Y}}{\left(u^{2}\right)^{2}} \gamma^{\beta} u_{\beta} u^{\alpha}+\frac{(\widetilde{Y} L \tilde{Y})^{2}}{4 G\left(u^{2}\right)^{3}}\left(2 \gamma^{\beta} u^{\alpha}-\gamma^{\alpha} u^{\beta}\right) u_{\beta}\right) \partial_{\alpha} \theta,
\end{aligned}
$$

which gives

$$
U^{\alpha}=G \gamma^{\alpha}+\frac{\tilde{Y} M \tilde{Y}}{\left(u^{2}\right)^{2}} \gamma^{\beta} u_{\beta} u^{\alpha}+\frac{(\tilde{Y} L \tilde{Y})^{2}}{4 G\left(u^{2}\right)^{3}}\left(2 \gamma^{\beta} u^{\alpha}-\gamma^{\alpha} u^{\beta}\right) u_{\beta}
$$

\section{A.3. Evaluation of $T^{\alpha}$}

First let us evaluate $\delta \mathcal{L}_{2}$ :

$$
\begin{aligned}
\delta \mathcal{L}_{2} & =-\frac{\tilde{Y} L \partial_{\beta} Y u_{\alpha}}{2 u^{2}}\left(\delta G^{\beta \alpha}\right)-\frac{\tilde{Y} L \partial_{\beta} Y u^{\beta}}{2} \delta\left(\frac{1}{u^{2}}\right) \\
& =\delta \bar{\theta}\left(-\frac{\tilde{Y} L \partial_{\beta} Y u_{\alpha} \gamma^{\{\beta} \partial^{\alpha\}} \theta}{u^{2}}+\frac{2 \tilde{Y} L \partial_{\beta} Y u^{\beta} \gamma^{\gamma} \partial^{\alpha} \theta u_{\gamma} u_{\alpha}}{\left(u^{2}\right)^{2}}\right) \\
& =\delta \bar{\theta} \frac{\tilde{Y} L}{\left(u^{2}\right)^{2}}\left(-\partial_{\beta} Y \gamma^{\{\beta} G^{\gamma\} \alpha} u_{\gamma} u^{2}+2 \partial_{\beta} Y u^{\beta} \gamma^{\gamma} u_{\gamma} u^{\alpha}\right) \partial_{\alpha} \theta
\end{aligned}
$$

This can be simplified more by rewriting the second term in the parenthesis as

$$
\begin{aligned}
2 \partial_{\beta} Y u^{\beta} u_{\gamma} \gamma^{\gamma} u^{\alpha} & =\partial_{\gamma} Y u^{\gamma} u_{\beta}\left(\gamma^{\beta} u^{\alpha}+\gamma^{\delta} u_{\delta} G^{\beta \alpha}\right) \\
& =\left(\partial_{\beta} Y u^{2}+\widetilde{Y} \epsilon_{\beta \gamma} u^{\gamma}\right)\left(\gamma^{\beta} u^{\alpha}+\gamma^{\delta} u_{\delta} G^{\beta \alpha}\right) \\
& =\partial_{\beta} Y \gamma^{\{\beta} G^{\gamma\} \alpha} u_{\gamma} u^{2}+\widetilde{Y} \epsilon_{\beta \gamma} u^{\gamma}\left(\gamma^{\beta} u^{\alpha}+\gamma^{\delta} u_{\delta} G^{\beta \alpha}\right),
\end{aligned}
$$

where we have used

$$
\partial_{\gamma} Y u^{\gamma} u_{\beta}=\partial_{\beta} Y u^{2}+\widetilde{Y} \epsilon_{\beta \gamma} u^{\gamma}
$$


Then this cancels out the first term of $\delta \mathcal{L}_{2}$ giving

$$
\begin{aligned}
\delta \mathcal{L}_{2} & =\delta \bar{\theta} \frac{\tilde{Y} L \tilde{Y}}{\left(u^{2}\right)^{2}} \epsilon_{\beta \gamma} u^{\gamma}\left(\gamma^{\beta} u^{\alpha}+\gamma^{\delta} u_{\delta} G^{\beta \alpha}\right) \partial_{\alpha} \theta \\
& =2 \delta \bar{\theta}\left(\frac{\tilde{Y} L \tilde{Y}}{2\left(u^{2}\right)^{2}} \epsilon_{\beta \gamma} \gamma^{\{\beta} G^{\delta\} \alpha} u^{\gamma} u_{\delta}\right) \partial_{\alpha} \theta
\end{aligned}
$$

Now let us evaluate $\delta \mathcal{L}_{3}$,

$$
\delta \mathcal{L}_{3}=-\epsilon^{\alpha \beta} \delta\left(\bar{\theta} \Gamma_{m} \partial_{\alpha} \theta\right) \partial_{\beta} X^{m}-\epsilon^{\alpha \beta}\left(\bar{\theta} \Gamma_{m} \partial_{\alpha} \theta\right) \partial_{\beta} \delta X^{m}
$$

Here the first term becomes

$$
\begin{aligned}
-\epsilon^{\alpha \beta} \delta\left(\bar{\theta} \Gamma_{m} \partial_{\alpha} \theta\right) \partial_{\beta} X^{m} & =-\epsilon^{\alpha \beta}\left(\delta \bar{\theta} \Gamma_{m} \partial_{\alpha} \theta+\bar{\theta} \Gamma_{m} \partial_{\alpha} \delta \theta\right) \partial_{\beta} X^{m} \\
& =-\epsilon^{\alpha \beta}\left(\delta \bar{\theta} \Gamma_{m} \partial_{\alpha} \theta-\partial_{\alpha} \delta \bar{\theta} \Gamma_{m} \theta\right) \partial_{\beta} X^{m} \\
& =-\epsilon^{\alpha \beta}\left(2 \delta \bar{\theta} \Gamma_{m} \partial_{\alpha} \theta\right) \partial_{\beta} X^{m}-\underbrace{\epsilon^{\alpha \beta} \partial_{\alpha}\left(\delta \bar{\theta} \Gamma_{m} \theta\right) \partial_{\beta} X^{m}}_{\text {total derivative }},
\end{aligned}
$$

where the second equality is realized since $\theta$ is Majorana. Next, the second term becomes

$$
\begin{aligned}
-\epsilon^{\alpha \beta}\left(\bar{\theta} \Gamma_{m} \partial_{\alpha} \theta\right) \partial_{\beta} \delta X^{m} & =\epsilon^{\alpha \beta} \partial_{\alpha} \delta X_{m}\left(\bar{\theta} \Gamma^{m} \partial_{\beta} \theta\right) \\
& =\epsilon^{\alpha \beta} \partial_{\alpha}\left(-\delta \bar{\theta} \Gamma_{m} \theta\right)\left(\bar{\theta} \Gamma^{m} \partial_{\beta} \theta\right) \\
& =\epsilon^{\alpha \beta}\left(\delta \bar{\theta} \Gamma_{m} \theta\right)\left(\partial_{\alpha} \bar{\theta} \Gamma^{m} \partial_{\beta} \theta\right)+\underbrace{\epsilon^{\alpha \beta} \partial_{\alpha}\left(-\delta \bar{\theta} \Gamma_{m} \theta \bar{\theta}\right) \Gamma^{m} \partial_{\beta} \theta}_{\text {total derivative }} .
\end{aligned}
$$

Moreover, we can use a spinor identity [20], which is realized for Majorana-Weyl spinors in 10-d, to change the last line as

$$
\epsilon^{\alpha \beta}\left(\delta \bar{\theta} \Gamma_{m} \theta\right)\left(\partial_{\alpha} \bar{\theta} \Gamma^{m} \partial_{\beta} \theta\right)=2 \epsilon^{\alpha \beta}\left(\delta \bar{\theta} \Gamma_{m} \partial_{\alpha} \theta\right)\left(\bar{\theta} \Gamma^{m} \partial_{\beta} \theta\right)
$$

Finally, collecting the two terms of $\delta \mathcal{L}_{3}$ again,

$$
\begin{aligned}
\delta \mathcal{L}_{3} & =-2 \epsilon^{\alpha \beta}\left(\delta \bar{\theta} \Gamma_{m} \partial_{\alpha} \theta\right)\left(\partial_{\beta} X^{m}-\bar{\theta} \Gamma^{m} \partial_{\beta} \theta\right) \\
& =-2 \epsilon^{\alpha \beta}\left(\delta \bar{\theta} \Gamma_{m} \partial_{\alpha} \theta\right) \Pi_{\beta}^{m} \\
& =2 \delta \bar{\theta}\left(-\epsilon^{\alpha \beta} \Gamma_{m} \Pi_{\beta}^{m}\right) \partial_{\alpha} \theta \\
& =2 \delta \bar{\theta}\left(-\epsilon^{\alpha \beta} \gamma_{\beta}\right) \partial_{\alpha} \theta \\
& =2 \delta \bar{\theta}\left(-\bar{\gamma} \gamma^{\alpha}\right) \partial_{\alpha} \theta
\end{aligned}
$$

In result, we get $T^{\alpha}$ from $\delta \mathcal{L}_{2}+\delta \mathcal{L}_{3}$ as

$$
T^{\alpha}=-\bar{\gamma} \gamma^{\alpha}+\frac{\tilde{Y} L \tilde{Y}}{2\left(u^{2}\right)^{2}} \epsilon_{\beta \gamma} \gamma^{\{\beta} G^{\delta\} \alpha} u^{\gamma} u_{\delta} .
$$




\section{A.4. Determination of $\rho$}

Equipped with $U^{\alpha}$ and $T^{\alpha}$, we now find an appropriate $\rho$ with $\rho^{2}=\left(\mathcal{L}_{1}\right)^{2}$ and show $U^{\alpha}=\rho T^{\alpha}$ to complete the proof. First, we take $\rho$ as

$$
\rho=\bar{\gamma}-\frac{\widetilde{Y} L \widetilde{Y}}{2 G u^{2}} \bar{\gamma}
$$

Then $\rho^{2}$ becomes

$$
\begin{aligned}
\rho^{2} & =\bar{\gamma}^{2}-\frac{\tilde{Y} L \tilde{Y}}{G u^{2}} \bar{\gamma}^{2}+\frac{(\tilde{Y} L \tilde{Y})^{2}}{4 G^{2}\left(u^{2}\right)^{2}} \bar{\gamma}^{2} \\
& =-G-\frac{\tilde{Y} M \tilde{Y}}{u^{2}}-\frac{(\widetilde{Y} L \tilde{Y})^{2}}{4 G\left(u^{2}\right)^{2}} \\
& =\left(\mathcal{L}_{1}\right)^{2}
\end{aligned}
$$

where $\bar{\gamma}^{2}=-G$ and $M=-L$ have been used.

\section{A.5. Proof of $U^{\alpha}=\rho T^{\alpha}$}

To show $U^{\alpha}=\rho T^{\alpha}$, we decompose $U^{\alpha}, T^{\alpha}$ and $\rho$ according to the power of $\tilde{Y}^{I}$ as

$$
\begin{aligned}
& U_{0}^{\alpha}=G \gamma^{\alpha}, \\
& U_{1}^{\alpha}=0, \\
& U_{2}^{\alpha}= \frac{\widetilde{Y} M \widetilde{Y}}{\left(u^{2}\right)^{2}} \gamma^{\beta} u_{\beta} u^{\alpha}, \\
& U_{3}^{\alpha}= 0, \\
& U_{4}^{\alpha}= \frac{(\widetilde{Y} L \tilde{Y})^{2}}{4 G\left(u^{2}\right)^{3}}\left(2 \gamma^{\beta} u^{\alpha}-\gamma^{\alpha} u^{\beta}\right) u_{\beta}, \\
& T_{0}^{\alpha}=-\bar{\gamma} \gamma^{\alpha}, \\
& T_{1}^{\alpha}=0, \\
& T_{2}^{\alpha}=\frac{\tilde{Y} L \tilde{Y}}{2\left(u^{2}\right)^{2}} \epsilon_{\beta \gamma} \gamma^{\{\beta} G^{\delta\} \alpha} u^{\gamma} u_{\delta}, \\
& \rho_{0}=\bar{\gamma}, \\
& \rho_{1}=0, \\
& \rho_{2}=-\frac{Y}{2 G u^{2}} \bar{\gamma},
\end{aligned}
$$


where the subscripts of $U, T$ and $\rho$ denote the powers of $\tilde{Y}$. Then $U^{\alpha}=\rho T^{\alpha}$ becomes

$$
\begin{aligned}
& U_{0}^{\alpha}=\rho_{0} T_{0}^{\alpha}, \\
& U_{1}^{\alpha}=\rho_{0} T_{1}^{\alpha}+\rho_{1} T_{0}^{\alpha}, \\
& U_{2}^{\alpha}=\rho_{0} T_{2}^{\alpha}+\rho_{1} T_{1}^{\alpha}+\rho_{2} T_{0}^{\alpha}, \\
& U_{3}^{\alpha}=\rho_{1} T_{2}^{\alpha}+\rho_{2} T_{1}^{\alpha}, \\
& U_{4}^{\alpha}=\rho_{2} T_{2}^{\alpha} .
\end{aligned}
$$

Then we can complete the proof of kappa symmetry by checking above relations. First $U_{1}^{\alpha}=\rho_{0} T_{1}^{\alpha}+\rho_{1} T_{0}^{\alpha}$ and $U_{3}^{\alpha}=\rho_{1} T_{2}^{\alpha}+\rho_{2} T_{1}^{\alpha}$ are checked easily, since $T_{1}^{\alpha}=\rho_{1}=0$ and $U_{1}^{\alpha}=U_{3}^{\alpha}=0$.

$$
U_{0}^{\alpha}=\rho_{0} T_{1}^{\alpha}+\rho_{1} T_{0}^{\alpha} \text { is checked as }
$$

$$
\rho_{0} T_{0}^{\alpha}=-\bar{\gamma}^{2} \gamma^{\alpha}=G \gamma^{\alpha}=U_{0}^{\alpha},
$$

where we have used $\bar{\gamma}^{2}=-G$.

Next see how $U_{2}^{\alpha}=\rho_{0} T_{2}^{\alpha}+\rho_{1} T_{1}^{\alpha}+\rho_{2} T_{0}^{\alpha}$ is realized. First $\rho_{0} T_{2}^{\alpha}$ is evaluated as, omitting the factor $\frac{\widetilde{Y} L \widetilde{Y}}{2\left(u^{2}\right)^{2}} u^{\gamma} u_{\delta}$

$$
\begin{aligned}
\rho_{0} T_{2}^{\alpha} & \sim \bar{\gamma} \epsilon_{\beta \gamma} \gamma^{\{\beta} G^{\delta\} \alpha} \\
& =\gamma_{\beta \gamma} \gamma^{\{\beta} G^{\delta\} \alpha} \\
& =\gamma_{\beta \gamma} \gamma_{\epsilon} G^{\epsilon\{\beta} G^{\delta\} \alpha} \\
& =\gamma_{[\beta} G_{\gamma] \epsilon} G^{\epsilon\{\beta} G^{\delta\} \alpha} \\
& =\gamma_{[\beta} \delta_{\gamma]}^{\{\beta} G^{\delta\} \alpha} \\
& =\left(\gamma_{\gamma}-2 \gamma_{\gamma}\right) G^{\delta \alpha}+\gamma^{\alpha} \delta_{\gamma}^{\delta}-\gamma_{\gamma} G^{\delta \alpha} \\
& =-2 \gamma_{\gamma} G^{\delta \alpha}+\delta_{\gamma}^{\delta} \gamma^{\alpha},
\end{aligned}
$$

where we used $\bar{\gamma} \epsilon_{\beta \gamma}=\gamma_{\beta \gamma}$ and $\gamma_{\beta \gamma} \gamma_{\epsilon}=\gamma_{[\beta} G_{\gamma] \epsilon}$. Then,

$$
\begin{aligned}
\rho_{0} T_{2}^{\alpha} & =\frac{\tilde{Y} L \tilde{Y}}{2\left(u^{2}\right)^{2}} u^{\gamma} u_{\delta}\left(-2 \gamma_{\gamma} G^{\delta \alpha}+\delta_{\gamma}^{\delta} \gamma^{\alpha}\right) \\
& =\frac{\widetilde{Y} L \tilde{Y}}{2\left(u^{2}\right)^{2}}\left(-2 \gamma^{\beta} u^{\alpha}+\gamma^{\alpha} u^{\beta}\right) u_{\beta} .
\end{aligned}
$$

Secondly, $\rho_{1} T_{1}^{\alpha}=0$ from $\rho_{1}=0$. Lastly,

$$
\begin{aligned}
\rho_{2} T_{0}^{\alpha} & =\frac{\widetilde{Y} L \tilde{Y}}{2 G u^{2}} \bar{\gamma} \bar{\gamma} \gamma^{\alpha} \\
& =-\frac{\widetilde{Y} L \widetilde{Y}}{2\left(u^{2}\right)^{2}} \gamma^{\alpha} u^{\beta} u_{\beta},
\end{aligned}
$$


where $\bar{\gamma}^{2}=-G$ has been used. Then using $M=-L$,

$$
\begin{aligned}
\rho_{0} T_{2}^{\alpha}+\rho_{1} T_{1}^{\alpha}+\rho_{2} T_{0}^{\alpha} & =\frac{\widetilde{Y} M \tilde{Y}}{\left(u^{2}\right)^{2}} \gamma^{\beta} u_{\beta} u^{\alpha} \\
& =U_{2}^{\alpha} .
\end{aligned}
$$

Finally, $U_{4}^{\alpha}=\rho_{2} T_{2}^{\alpha}$ is proved as

$$
\begin{aligned}
\rho_{2} T_{2}^{\alpha} & =-\frac{\tilde{Y} L \tilde{Y}}{2 G u^{2}} \rho_{0} T_{2}^{\alpha} \\
& =\frac{(\widetilde{Y} L \widetilde{Y})^{2}}{4 G\left(u^{2}\right)^{3}}\left(2 \gamma^{\beta} u^{\alpha}-\gamma^{\alpha} u^{\beta}\right) u_{\beta} \\
& =U_{4}^{\alpha} .
\end{aligned}
$$

Thus we have proved the kappa symmetry of 10-d heterotic action.

\section{References}

[1] S. Cherkis and J. H. Schwarz, "Wrapping the M Theory Five-Brane on K3", Phys. Lett. B403 (1997) 225 arXiv:hep-th/9703062.

[2] M. Perry and J. H. Schwarz, "Interacting Chiral Gauge Fields in Six Dimensions and Born-Infeld Theory", Nucl. Phys. B489 (1997) 47 [arXiv:hep-th/9611065].

[3] P. Pasti, D. Sorokin, and M. Tonin, "Covariant Action for a $\mathrm{D}=11$ Five-Brane with the Chiral Field", Phys. Lett. B398 (1997) 41 [arXiv:hep-th/9701037.

[4] M. Aganagic, J. Park, C. Popescu, and J. H. Schwarz, "World-Volume Action of the M Theory Five-Brane", Nucl. Phys. B496 (1997) 191 [arXiv:hep-th/9701166].

[5] I. Bandos, K. Lechner, A. Nurmagambetov, P. Pasti, D. Sorokin, and M. Tonin, "Covariant Action for the Super-Five-Brane of M-Theory", Phys. Rev. Lett. 78 (1997) 4332 arXiv:hep-th/9701149].

[6] I. Bandos, K. Lechner, A. Nurmagambetov, P. Pasti, D. Sorokin, and M. Tonin, "On the Equivalence of Different Formulations of the M Theory Five-Brane", Phys. Lett. B408 (1997) 135 arXiv:hep-th/9703127.

[7] P. S. Howe, E. Sezgin, and P. C. West, "Covariant Field Equations of the M-Theory Five-Brane", Phys. Lett. B399 (1997) 49 [arXiv:hep-th/9702008].

[8] D. P. Sorokin, "Superbranes and Superembeddings", Phys. Rept. 329 (2000) 1 arXiv:hep-th/9906142.

[9] M. Tonin, " $\kappa$ Symmetry as World Sheet Supersymmetry in D=10 Heterotic Superstring", International Journal of Modern Physics A7 (1992) 6013.

[10] M. Tonin, "Twistor-Like Formulation of Heterotic Strings", Talk given at the X Italian Conference on General Relativity and Gravitational Physics - Bardonecchia (1992) arXiv:hep-th/9301055. 
[11] A. Candiello, K. Lechner, and M. Tonin, "k-Anomalies and Space-Time Supersymmetry in the Green-Schwarz Heterotic Superstring", Nucl. Phys. B438 (1995) 67 arXiv:hep-th/9409107.

[12] M. Cederwall, "Boundaries of 11-Dimensional Membranes", Mod. Phys. Lett. A12 (1997) 2641 arXiv:hep-th/9704161.

[13] J. P. Serre, "A Course in Arithmetic", Springer-Verlag (1973).

[14] J. H. Conway and N. J. A. Sloane, "Sphere Packings, Lattices, and Groups", SpringerVerlag (1993).

[15] P. S. Aspinwall, "K3 Surfaces and String Duality" arXiv:hep-th/9611137.

[16] B. de Wit, K. Peeters, and J. Plefka, "Superspace Geometry for Supermembrane Backgrounds", Nucl. Phys. B532 (1998) 99 arXiv:hep-th/9803209].

[17] R. Kallosh and D. Sorokin, "Dirac Action on M5 and M2 Branes with Bulk Fluxes", JHEP 0505 (2005) 005 arXiv:hep-th/0501081.

[18] T. Kugo and P. Townsend, "Supersymmetry and Division Algebras", Nucl. Phys. B221 (1983) 357.

[19] A. F. Kleppe and C. Wainwright, "Graded Majorana Spinors", J. Phys. A: Math. Gen. 39 (2006) 3787.

[20] M. B. Green, J. H. Schwarz, and E. Witten, "Superstring Theory" Vol. 2, Cambridge University Press (1987).

[21] P. Pasti, D. Sorokin, and M. Tonin, "On Lorentz Invariant Actions for Chiral P-Forms", Phys. Rev. D55 (1997) 6292 [arXiv:hep-th/9611100]. 